\title{
Hyperentanglement purification for two-photon six-qubit quantum systems*
}

\author{
Guan-Yu Wang, Qian Liu, and Fu-Guo Deng ${ }^{\dagger}$ \\ Department of Physics, Applied Optics Beijing Area Major Laboratory, \\ Beijing Normal University, Beijing 100875, China
}

(Dated: October 2, 2018)

\begin{abstract}
Recently, two-photon six-qubit hyperentangled states were produced in experiment and they can improve the channel capacity of quantum communication largely. Here we present a scheme for the hyperentanglement purification of nonlocal two-photon systems in three degrees of freedom (DOFs), including the polarization, the first-longitudinal-momentum, and the second longitudinal momentum DOFs. Our hyperentanglement purification protocol (hyper-EPP) is constructed with two steps resorting to parity-check quantum nondemolition measurement on the three DOFs and SWAP gates, respectively. With these two steps, the bit-flip errors in the three DOFs can be corrected efficiently. Also, we show that using SWAP gates is a universal method for hyper-EPP in the polarization DOF and multiple longitudinal momentum DOFs. The implementation of our hyper-EPP is assisted by nitrogen-vacancy centers in optical microcavities, which could be achieved with current techniques. It is useful for long-distance high-capacity quantum communication with two-photon six-qubit hyperentanglement.
\end{abstract}

PACS numbers: 03.67.Bg, 03.67.Pp, 03.65.Yz, 03.67.Hk

\section{INTRODUCTION}

Quantum entanglement plays a critical role in quantum information processing [1]. It is the key resource in quantum communication, such as quantum teleportation [2], quantum dense coding [3, 4], quantum key distribution [5, 6], quantum secret sharing [], quantum secure direct communication [8, 9] , and so on. Maximally entangled states can be used as the quantum channel for teleporting an unknown state of a quantum particle, without moving the particle itself [2]. It can also be used to carry two bits of information by moving only one two-level particle [3], not two or more. The two parties of quantum communication can create a private key with entangled photon pairs in an absolutely secure way [ $[, 6]$. Moreover, they can exchange the secret message directly and securely without distributing the private key if they share maximally entangled photon pairs [8, 9].

Hyperentanglement, a state of a quantum system being simultaneously entangled in multiple degrees of freedom (DOFs), has attracted much attention as it can improve both the channel capacity and the security of quantum communication largely, beat the channel capacity of linear photonic superdense coding [10], assist the complete analysis of Bell states [11 14, and so on. With the $\beta$ barium borate (BBO) crystal, photon pairs produced by spontaneous parametric down conversion (PDC) can be in a hyperentangled state. Many theoretical and experimental schemes for the generation of hyperentangled states have been proposed and implemented in optical systems [15 23], such as in polarization-momentum DOFs [18], polarization-orbital-

\footnotetext{
*Published in Phys. Rev. A 94, 032319 (2016)

†Corresponding author: fgdeng@bnu.edu.cn
}

angular momentum DOFs [19], multipath DOFs [20], and so on. In 2009, Vallone et al. 23] demonstrated experimentally the generation of a two-photon six-qubit hyperentangled state in three DOFs.

Although the interaction between a photon and its environment is weaker than other quantum systems, it still inevitably suffers from channel noise, such as thermal fluctuation, vibration, imperfection of an optical fiber, and birefringence effects. The interaction between the photons and the environment will make the entangled photon pairs in less entangled states or even in mixed states, which will decrease the security and the efficiency of quantum communication. Entanglement purification is used to obtain a subset of high-fidelity nonlocal entangled quantum systems from a set of those in mixed entangled states 24 28]. In 1996, Bennett et al. 24] introduced the entanglement purification protocol (EPP) for quantum systems in a Werner state 29] with quantum controlled-NOT gates. In 2001, Pan et al. [26] proposed an EPP with linear optical elements. In 2002, Simon and Pan [27] presented an EPP for a PDC source, not an ideal entanglement source. In 2008, Sheng et al. 28] proposed an efficient EPP for polarization entanglement from a PDC source, assisted by nondestructive quantum nondemolition detectors (QND) with cross-Kerr nonlinearity. In 2010, Sheng and Deng [30] introduced the original deterministic EPP for two-photon systems, which is far different from the conventional EPPs [24 28] as it works in a deterministic way, not a probabilistic one. Subsequently, some interesting deterministic EPPs were proposed [31 34]. In 2003, Pan et al. 35] demonstrated the entanglement purification of photon pairs using linear optical elements. Recently, Ren and Deng 36] proposed the original hyperentanglement purification protocol (hyper-EPP) for two-photon four-qubit systems in mixed polarization-spatial hyperentangled Bell states with polarization bit-flip errors and spatial-mode bit-flip 
errors assisted by nonlinear optical elements.

A nitrogen vacancy (NV) center in diamond is an attractive candidate for quantum information processing because of its long-lived coherence time at room temperature and optical controllability. The coherence time of a diamond NV center can continue for $1.8 \mathrm{~ms}$ [37]. In a diamond NV center, the electron spin can be exactly populated by the optical pumping with 532-nm light, and it can be easily manipulated [38-41] and readout [42, 43] by using the microwave excitation. Many interesting approaches based on an NV center in diamond coupled to an optical cavity have been proposed in theory [44 48] and implemented in experiment [49 53 ].

In this paper, we present a hyper-EPP for nonlocal photon systems entangled in three DOFs assisted by nitrogen-vacancy centers in optical microcavities, including the polarization DOF, the first-longitudinal-momentum DOF, and the secondlongitudinal-momentum DOF. Our protocol is completed by two steps. The first step resorts to parity-check quantum nondemolition measurement on the polarization DOF (P-QND) and the two longitudinal-momentum DOFs (S-QND). The second step resorts to the SWAP gate between the polarization states of two photons (PP-SWAP gate) and the SWAP gate between the polarization state and the spatial state of one photon (P-F-SWAP gate and P-S-SWAP gate). Also, we show that using the SWAP gates is a universal method for hyper-EPP in the polarization DOF and multiple longitudinal-momentum DOFs. Our hyper-EPP can effectively improve the entanglement of photon systems in long-distance quantum communication.

This paper is organized as follows: We give the interface between a circularly polarized light and a diamond nitrogen-vacancy center confined in an optical microcavity in Sec. III Subsequently, we present the polarizationspatial parity-check QND of two-photon six-qubit systems in Sec. III and then, we give the principle of our SWAP gate between the polarization states of two photons in Sec. IVA and that of our SWAP gate between the polarization state and the spatial state of one photon in Sec. IVB In SecV we propose an efficient hyperEPP for mixed two-photon six-qubit hyperentangled Bell states. In Sec. VI, we discuss the expansion for purifying the two-photon systems in the polarization DOF and multiple longitudinal momentum DOFs. A discussion and a summary are given in Sec. VII In addition, some other cases for our hyper-EPP are discussed in the Appendix.

\section{THE INTERFACE BETWEEN A CIRCULARLY POLARIZED LIGHT AND A DIAMOND NITROGEN-VACANCY CENTER CONFINED IN AN OPTICAL MICROCAVITY}

A diamond NV center consists of a vacancy adjacent to a substitutional nitrogen atom. The NV center is neg- atively charged with two unpaired electrons located at the vacancy. The energy-level structure of the NV center coupled to the cavity mode is shown in Fig. 1(a). The ground state is a spin triplet with the splitting at 2.87 $\mathrm{GHz}$ in a zero external field between levels $|0\rangle \equiv|m=0\rangle$ and $| \pm 1\rangle \equiv|m= \pm 1\rangle$ owing to spin-spin interaction. The excited state, which is one of the six eigenstates of the full Hamiltonian including spin-spin and spin-orbit interactions in the absence of any perturbation, is labeled as $\left|A_{2}\right\rangle=\left|E_{-}\right\rangle|+1\rangle+\left|E_{+}\right\rangle|-1\rangle$, where $\left|E_{+}\right\rangle$and $\left|E_{-}\right\rangle$ are the orbital states with the angular momentum projections +1 and -1 along the $\mathrm{NV}$ axis, respectively. We encode the qubits on the sublevels $| \pm 1\rangle$ as the ground states, and take $\left|A_{2}\right\rangle$ as an auxiliary excited state. The transitions $|+1\rangle \leftrightarrow\left|A_{2}\right\rangle$ and $|-1\rangle \leftrightarrow\left|A_{2}\right\rangle$ in the NV center are resonantly coupled to the right $(\mathrm{R})$ and the left (L) circularly polarized photons with the identical transition frequency, respectively. The two transitions take place with equal probability.

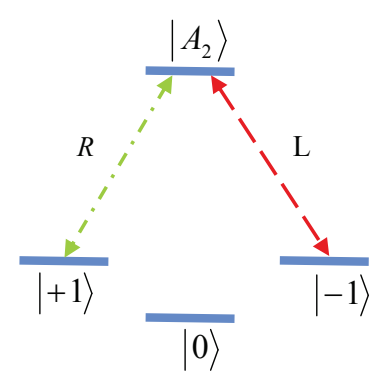

(a)

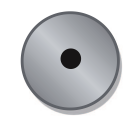

(b)
FIG. 1: (a) The energy-level diagram of an NV-cavity system. The qubit is encoded on the ground states $| \pm 1\rangle$ and the excited state $\left|A_{2}\right\rangle$. The transitions $| \pm 1\rangle \leftrightarrow\left|A_{2}\right\rangle$ are driven by the right and light circularly polarized photons, respectively. (b) Schematic diagram for an NV center confined inside a single-sided optical resonant microcavity.

Let us consider the composite unit, a diamond NV center confined inside a single-sided resonant microcavity, as shown in Fig. 1(b). The Heisenberg equations of motion for this system can be written as 54]

$$
\begin{aligned}
\dot{a}(t)= & -\left[i\left(\omega_{c}-\omega_{p}\right)+\frac{\kappa}{2}\right] a(t)-g \sigma_{-}(t) \\
& -\sqrt{\kappa} a_{\text {in }}, \\
\dot{\sigma}_{-}(t)= & -\left[i\left(\omega_{0}-\omega_{p}\right)+\frac{\gamma}{2}\right] \sigma_{-}(t)-g \sigma_{z}(t) a(t) \\
& +\sqrt{\gamma} \sigma_{z}(t) b_{\text {in }}(t), \\
a_{\text {out }}(t)= & a_{\text {in }}(t)+\sqrt{\kappa} a(t) .
\end{aligned}
$$

Here $a(t)$ and $\sigma_{-}(t)$ are the annihilation operator of the cavity mode and the transition operator of the diamond NV center. $\sigma_{z}(t)$ represents the inversion operator of the NV center. $\omega_{c}, \omega_{p}$, and $\omega_{0}$ are the frequencies of the cavity mode, the photon, and the diamond NV center 
level transition, respectively. $g, \gamma$, and $\kappa$ are the coupling strength between a diamond NV center and a cavity, the decay rate of a diamond NV center, and the damping rate of a cavity, respectively. $b_{i n}(t)$ is the vacuum input field with the commutation relation $\left[b_{i n}(t), b_{i n}^{\dagger}\left(t^{\prime}\right)\right]=\delta\left(t-t^{\prime}\right)$.

In the weak excitation limit, $\left\langle\sigma_{z}\right\rangle=-1$, the reflection coefficient for the NV-cavity unit is 55,56 .

$$
r\left(\omega_{p}\right)=\frac{\left[i\left(\omega_{c}-\omega_{p}\right)-\frac{\kappa}{2}\right]\left[i\left(\omega_{0}-\omega_{p}\right)+\frac{\gamma}{2}\right]+g^{2}}{\left[i\left(\omega_{c}-\omega_{p}\right)+\frac{\kappa}{2}\right]\left[i\left(\omega_{0}-\omega_{p}\right)+\frac{\gamma}{2}\right]+g^{2}}
$$

When the diamond NV center is uncoupled to the cavity or coupled to an empty cavity, that is, $g=0$, the reflection coefficient can be written as

$$
r_{0}\left(\omega_{p}\right)=\frac{i\left(\omega_{c}-\omega_{p}\right)-\frac{\kappa}{2}}{i\left(\omega_{c}-\omega_{p}\right)+\frac{\kappa}{2}}
$$

If $\omega_{0}=\omega_{c}=\omega_{p}$, the reflection coefficients can be written as

$$
r=\frac{-\kappa \gamma+4 g^{2}}{\kappa \gamma+4 g^{2}}, \quad r_{0}=-1
$$

That is, when $\omega_{0}=\omega_{c}=\omega_{p}$, the change of the input photon can be summarized as

$$
\begin{array}{ll}
|R\rangle|+1\rangle \rightarrow r|R\rangle|+1\rangle, & |R\rangle|-1\rangle \rightarrow-|R\rangle|-1\rangle \\
|L\rangle|+1\rangle \rightarrow-|L\rangle|+1\rangle, & |L\rangle|-1\rangle \rightarrow r|L\rangle|-1\rangle .
\end{array}
$$

Considering the condition $g \geq 5 \sqrt{\kappa \gamma}$, we can obtain the approximate evolution of the system composed of a diamond NV center and a photon as follows [45]:

$$
\begin{aligned}
& |R\rangle|+1\rangle \rightarrow|R\rangle|+1\rangle, \quad|R\rangle|-1\rangle \rightarrow-|R\rangle|-1\rangle \\
& |L\rangle|+1\rangle \rightarrow-|L\rangle|+1\rangle, \quad|L\rangle|-1\rangle \rightarrow|L\rangle|-1\rangle .
\end{aligned}
$$

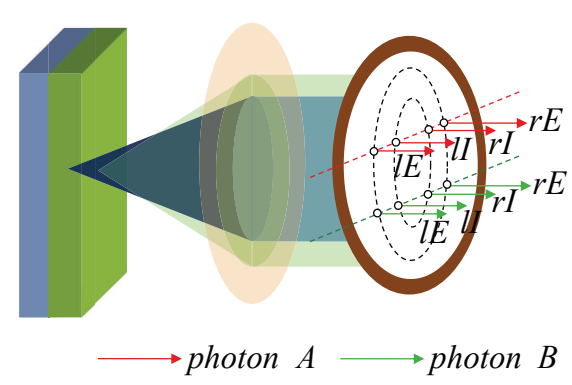

FIG. 2: The source of two-photon six-qubit hyperentanglement. The detailed description is shown in [23].

\section{POLARIZATION-SPATIAL PARITY-CHECK QND OF TWO-PHOTON SIX-QUBIT SYSTEMS}

A two-photon six-qubit hyperentangled Bell state can be described as follows:

$$
\begin{aligned}
|\Psi\rangle_{A B}= & \frac{1}{\sqrt{2}}\left(|H\rangle_{A}|H\rangle_{B}+|V\rangle_{A}|V\rangle_{B}\right) \\
& \otimes \frac{1}{\sqrt{2}}\left(|l\rangle_{A}|r\rangle_{B}+|r\rangle_{A}|l\rangle_{B}\right) \\
& \otimes \frac{1}{\sqrt{2}}\left(|I\rangle_{A}|I\rangle_{B}+|E\rangle_{A}|E\rangle_{B}\right) .
\end{aligned}
$$

Here the subscripts A and B represent the two photons. $H$ and $V$ represent the horizontal and the vertical polarizations of photons, respectively. $r$ and $l$ represent the right and the left spatial modes of a photon in the first-longitudinal-momentum DOF, respectively. $E$ and $I$ denote the external and the internal spatial modes of a photon in the second-longitudinal-momentum DOF, respectively, shown in Fig. 2. This two-photon six-qubit hyperentangled Bell state can be produced by two 0.5mm-thick type I BBO crystal slabs aligning one behind the other and an eight-hole screen [23], shown in Fig. 2. When a continuous-wave (cw) vertically polarized $\mathrm{Ar}^{+}$laser beam interacts through spontaneous parametric downconversion (SPDC) with the two BBO crystal slabs, the nonlinear interaction between the laser beam and the BBO crystal leads to the production of the degenerate photon pairs which are entangled in polarization and belong to the surfaces of two emission cones. As shown in Fig. 2, the insertion of an eight-hole screen allows us to achieve the double longitudinal-momentum entanglement.

Generally speaking, an arbitrary two-photon six-qubit hyperentangled Bell state for a photon pair $A B$ can be written as

$$
\left|\Psi_{6}\right\rangle_{A B}=|\psi\rangle_{P} \otimes|\psi\rangle_{F} \otimes|\psi\rangle_{S}
$$

where $|\psi\rangle_{P}$ is one of the four Bell states for a two-photon system in the polarization DOF,

$$
\begin{aligned}
\left|\phi^{ \pm}\right\rangle^{p} & =\frac{1}{\sqrt{2}}(|R R\rangle \pm|L L\rangle)_{A C}, \\
\left|\psi^{ \pm}\right\rangle^{p} & =\frac{1}{\sqrt{2}}(|R L\rangle \pm|L R\rangle)_{A C} .
\end{aligned}
$$

$|\psi\rangle_{F}$ is one of the four Bell states for a two-photon system in the first-longitudinal-momentum DOF,

$$
\begin{aligned}
\left|\phi^{ \pm}\right\rangle^{F} & =\frac{1}{\sqrt{2}}(|r r\rangle \pm|l l\rangle)_{A C}, \\
\left|\psi^{ \pm}\right\rangle^{F} & =\frac{1}{\sqrt{2}}(|r l\rangle \pm|l r\rangle)_{A C},
\end{aligned}
$$

and $|\psi\rangle_{S}$ is one of the four Bell states for a two-photon 
system in the second-longitudinal-momentum DOF,

$$
\begin{aligned}
\left|\phi^{ \pm}\right\rangle^{S} & =\frac{1}{\sqrt{2}}(|E E\rangle \pm|I I\rangle)_{A C}, \\
\left|\psi^{ \pm}\right\rangle^{S} & =\frac{1}{\sqrt{2}}(|E I\rangle \pm|I E\rangle)_{A C} .
\end{aligned}
$$

The polarization-spatial parity-check QND is used to distinguish the odd-parity mode $\left(\left|\psi^{ \pm}\right\rangle_{p},\left|\psi^{ \pm}\right\rangle_{F}\right.$, and $\left.\left|\psi^{ \pm}\right\rangle_{S}\right)$ from the even-parity mode $\left(\left|\phi^{ \pm}\right\rangle_{p},\left|\phi^{ \pm}\right\rangle_{F}\right.$, and $\left.\left|\phi^{ \pm}\right\rangle_{S}\right)$ of the hyperentangled Bell states in both the polarization and the two longitudinal-momentum DOFs.

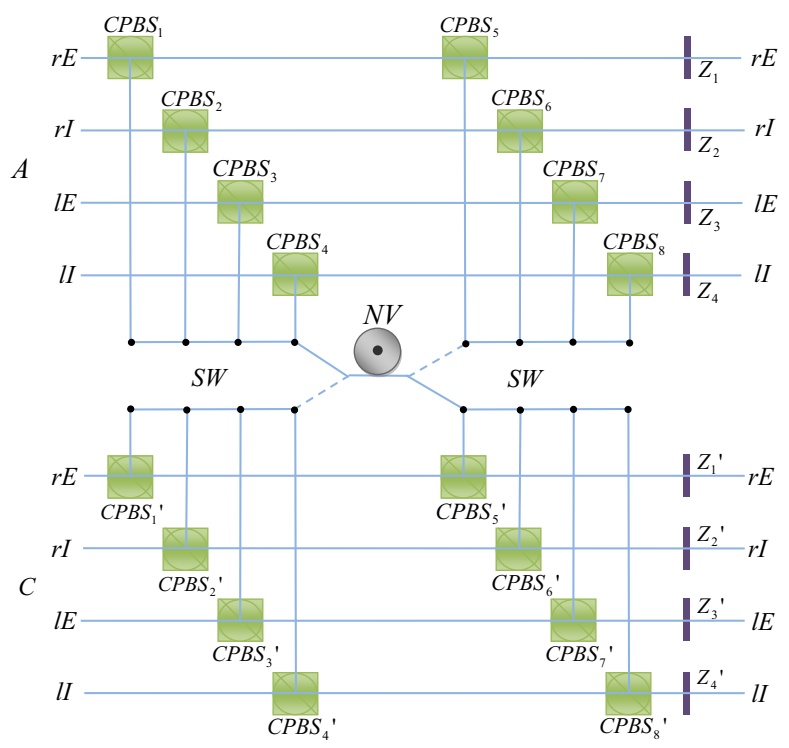

FIG. 3: Schematic diagram of the polarization parity-check QND. $C P B S_{i}\left(C P B S_{i^{\prime}}\right)(i=1,2,3,4)$ is a circularly polarizing beam splitter which transmits the photon in the rightcircular polarization $|R\rangle$ and reflects the photon in the leftcircular polarization $|L\rangle$, respectively. $Z_{i}\left(Z_{i^{\prime}}\right)(i=1,2,3,4)$ is a half-wave plate which performs a polarization phase-flip operation $\sigma_{z}^{P}=|R\rangle\langle R|-| L\rangle\langle L|$ on the photon. $S W$ is an optical switch which lets the wave packets of a photon in different spatial modes pass into and out of the NV center sequentially.

The schematic diagram of the polarization parity-check QND is shown in Fig. 3, which consists of some circularly polarizing beam splitters $\mathrm{CPBS}_{i}$, an NV center, and some half-wave plates $Z_{i}$. The $\mathrm{NV}$ center is prepared in the initial state $\left|\varphi^{+}\right\rangle=\frac{1}{\sqrt{2}}(|+1\rangle+|-1\rangle)$. If the two-photon system $A C$ is in a hyperentangled Bell state, one can inject the photons $A$ and $C$ into the quantum circuit sequentially. The evolutions of the system consisting of the two photons and the NV center are described as

$$
\begin{aligned}
\left|\phi^{ \pm}\right\rangle^{p}|\psi\rangle_{F}|\psi\rangle_{S} \otimes\left|\varphi^{+}\right\rangle & \rightarrow\left|\phi^{ \pm}\right\rangle^{p}|\psi\rangle_{F}|\psi\rangle_{S} \otimes\left|\varphi^{+}\right\rangle \\
\left|\psi^{ \pm}\right\rangle^{p}|\psi\rangle_{F}|\psi\rangle_{S} \otimes\left|\varphi^{+}\right\rangle & \rightarrow\left|\psi^{ \pm}\right\rangle^{p}|\psi\rangle_{F}|\psi\rangle_{S} \otimes\left|\varphi^{-}\right\rangle
\end{aligned}
$$

where $\left|\varphi^{-}\right\rangle=\frac{1}{\sqrt{2}}(|+1\rangle-|-1\rangle)$. By measuring the state of the NV center in the orthogonal basis $\left\{\left|\varphi^{+}\right\rangle,\left|\varphi^{-}\right\rangle\right\}$, one can distinguish the even-parity Bell states $\left|\phi^{ \pm}\right\rangle^{p}$ from the odd-parity Bell states $\left|\psi^{ \pm}\right\rangle^{p}$ of the two-photon system in the polarization DOF without affecting the states of the two-photon systems in the spatial-mode DOFs. That is, if the NV center is projected into the state $\left|\varphi^{+}\right\rangle$, the polarization state of the hyperentangled two-photon system is $\left|\phi^{ \pm}\right\rangle^{p}$. Otherwise, the polarization state of the hyperentangled two-photon system is $\left|\psi^{ \pm}\right\rangle^{p}$.

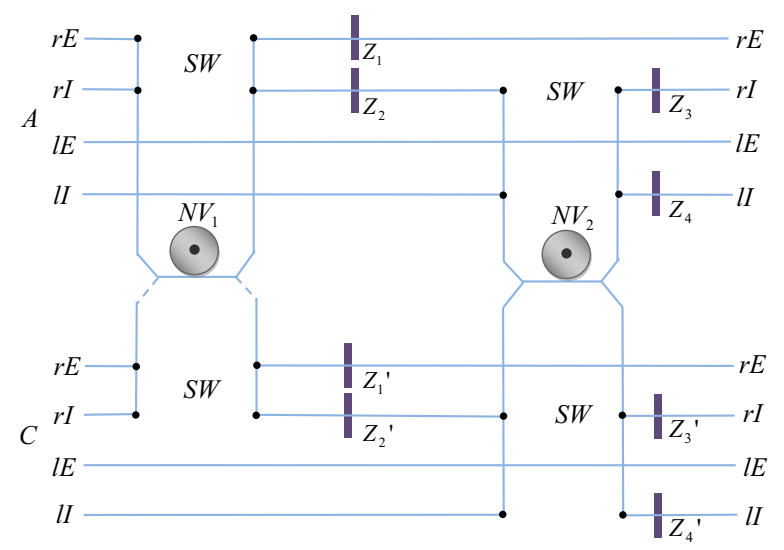

FIG. 4: Schematic diagram of the spatial-mode parity-check QND.

The schematic diagram of the spatial-mode paritycheck QND is shown in Fig. 4. It consists of two NV centers and some half-wave plates $Z_{i}\left(Z_{i^{\prime}}\right)$. Each of the two NV centers is prepared in the initial state $\left|\varphi^{+}\right\rangle_{i}=\frac{1}{\sqrt{2}}(|+1\rangle+|-1\rangle)$ with $i=1,2$. One can inject the photons $A$ and $C$ into the quantum circuit sequentially. The evolutions of the system composed of the two photons and the two NV centers are described as:

$$
\begin{aligned}
& |\psi\rangle_{P}\left|\phi^{ \pm}\right\rangle^{F}\left|\phi^{ \pm}\right\rangle^{S} \otimes\left|\varphi^{+}\right\rangle_{1}\left|\varphi^{+}\right\rangle_{2} \rightarrow|\psi\rangle_{P}\left|\phi^{ \pm}\right\rangle^{F}\left|\phi^{ \pm}\right\rangle^{S} \otimes\left|\varphi^{+}\right\rangle_{1}\left|\varphi^{+}\right\rangle_{2}, \\
& |\psi\rangle_{P}\left|\phi^{ \pm}\right\rangle^{F}\left|\psi^{ \pm}\right\rangle^{S} \otimes\left|\varphi^{+}\right\rangle_{1}\left|\varphi^{+}\right\rangle_{2} \rightarrow|\psi\rangle_{P}\left|\phi^{ \pm}\right\rangle^{F}\left|\psi^{ \pm}\right\rangle^{S} \otimes\left|\varphi^{+}\right\rangle_{1}\left|\varphi^{-}\right\rangle_{2}, \\
& |\psi\rangle_{P}\left|\psi^{ \pm}\right\rangle^{F}\left|\phi^{ \pm}\right\rangle^{S} \otimes\left|\varphi^{+}\right\rangle_{1}\left|\varphi^{+}\right\rangle_{2} \rightarrow|\psi\rangle_{P}\left|\psi^{ \pm}\right\rangle^{F}\left|\phi^{ \pm}\right\rangle^{S} \otimes\left|\varphi^{-}\right\rangle_{1}\left|\varphi^{+}\right\rangle_{2}, \\
& |\psi\rangle_{P}\left|\psi^{ \pm}\right\rangle^{F}\left|\psi^{ \pm}\right\rangle^{S} \otimes\left|\varphi^{+}\right\rangle_{1}\left|\varphi^{+}\right\rangle_{2} \rightarrow|\psi\rangle_{P}\left|\psi^{ \pm}\right\rangle^{F}\left|\psi^{ \pm}\right\rangle^{S} \otimes\left|\varphi^{-}\right\rangle_{1}\left|\varphi^{-}\right\rangle_{2} .
\end{aligned}
$$

By measuring the states of two NV centers in the orthogonal basis $\left\{\left|\varphi^{+}\right\rangle,\left|\varphi^{-}\right\rangle\right\}$, one can distinguish the evenparity states $\left|\phi^{ \pm}\right\rangle^{F}$ from the odd-parity states $\left|\psi^{ \pm}\right\rangle^{F}$ in the first-longitudinal-momentum DOF and the evenparity states $\left|\phi^{ \pm}\right\rangle^{S}$ from the odd-parity states $\left|\psi^{ \pm}\right\rangle^{S}$ in the second longitudinal momentum DOF, without affecting the states of the two-photon system in the polarization DOF. That is, if $N V_{1}$ is projected into the state $\left|\varphi^{+}\right\rangle_{1}$, the two-photon system is in the state $\left|\phi^{ \pm}\right\rangle^{F}$ in the first-longitudinal-momentum DOF and if $N V_{2}$ is projected into the state $\left|\varphi^{+}\right\rangle_{2}$, the two-photon system is in the state $\left|\phi^{ \pm}\right\rangle^{S}$ in the second-longitudinal-momentum DOF, respectively. If $N V_{1}$ is projected into the state $\left|\varphi^{-}\right\rangle_{1}$, the two-photon system is in the state $\left|\psi^{ \pm}\right\rangle^{F}$ in the first-longitudinal-momentum DOF and if $N V_{2}$ is projected into the state $\left|\varphi^{-}\right\rangle_{2}$, the two-photon system is 
in the state $\left|\psi^{ \pm}\right\rangle^{S}$ in the second-longitudinal-momentum DOF, respectively.

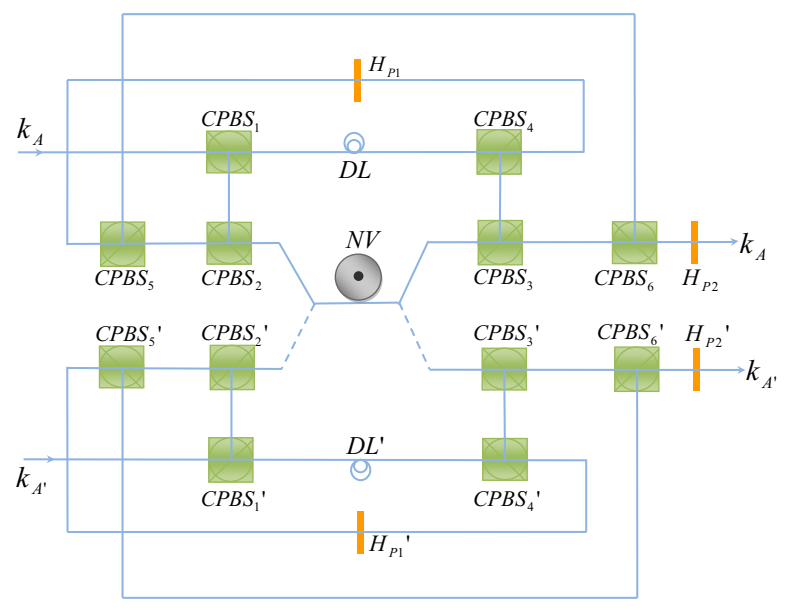

FIG. 5: Schematic diagram of the SWAP gate between the polarization states of photon $A$ and photon $A^{\prime}$ (P-P SWAP gate). $H_{p i}\left(i=1,2,1^{\prime}, 2^{\prime}\right)$ is a half-wave plate which performs the Hadamard operation $|R\rangle \rightarrow \frac{1}{\sqrt{2}}(|R\rangle+|L\rangle),|L\rangle \rightarrow$ $\frac{1}{\sqrt{2}}(|R\rangle-|L\rangle)$ on the polarization DOF of a photon. $D L$ $\left(D L^{\prime}\right)$ is a time-delay device which makes the two wave packets in different paths interfere with each other at last. $k$ $(k=r E, r I, l E, l I)$ represents any spatial modes of one photon.

\section{SWAP GATES}

\section{A. SWAP gate between the polarization states of two photons}

The SWAP gate between the polarization states of two photons (P-P-SWAP gate) is used to swap the polarization states of photon $A$ and photon $A^{\prime}$ without affecting their spatial-mode states. The initial states of two photons in the polarization DOF and two longitudinalmomentum DOFs are

$$
\begin{aligned}
& |\Phi\rangle_{A}=\left|\Phi_{p}\right\rangle_{A} \otimes\left|\Phi_{k}\right\rangle_{A}, \\
& |\Phi\rangle_{A^{\prime}}=\left|\Phi_{p}\right\rangle_{A^{\prime}} \otimes\left|\Phi_{k}\right\rangle_{A^{\prime}} .
\end{aligned}
$$

Here $\left|\Phi_{p}\right\rangle_{A}=\alpha_{1}|R\rangle+\beta_{1}|L\rangle$ and $\left|\Phi_{p}\right\rangle_{A^{\prime}}=\alpha_{2}|R\rangle+$ $\beta_{2}|L\rangle$ are the polarization states of the photons $A$ and $A^{\prime}$, respectively. $\quad\left|\Phi_{k}\right\rangle_{A}=\left(\gamma_{1}|r\rangle+\delta_{1}|l\rangle\right) \otimes$ $\left(\varepsilon_{1}|E\rangle+\xi_{1}|I\rangle\right)$ and $\left|\Phi_{k}\right\rangle_{A^{\prime}}=\left(\gamma_{2}|r\rangle+\delta_{2}|l\rangle\right) \otimes\left(\varepsilon_{2}|E\rangle+\right.$ $\left.\xi_{2}|I\rangle\right)$ represent the states of the photons $A$ and $A^{\prime}$ in both the first-longitudinal-momentum DOF and the second-longitudinal-momentum DOF, respectively. The schematic diagram of the P-P-SWAP gate is shown in Fig. 5. Suppose that the NV center is prepared in the initial state $|\Phi\rangle_{N V}=\frac{1}{\sqrt{2}}(|+1\rangle+|-1\rangle)$, the SWAP gate works with the following steps.
First, one injects the photons $A$ and $A^{\prime}$ into the quantum circuit sequentially, and lets photon $A\left(A^{\prime}\right)$ pass through the circularly polarizing beam splitter $C P B S_{1}$ $\left(C P B S_{1^{\prime}}\right), C P B S_{2}\left(C P B S_{2^{\prime}}\right)$, NV center, $C P B S_{3}$ $\left(C P B S_{3^{\prime}}\right)$, and $C P B S_{4}\left(C P B S_{4^{\prime}}\right)$ in sequence. After performing a Hadamard operation on the NV center $\left[|+1\rangle \rightarrow \frac{1}{\sqrt{2}}(|+1\rangle+|-1\rangle),|-1\rangle \rightarrow \frac{1}{\sqrt{2}}(|+1\rangle-|-1\rangle)\right]$, the whole state of the system composed of two photons and one NV center is transformed from $|\Phi\rangle_{0}$ to $|\Phi\rangle_{1}$. Here

$$
\begin{aligned}
|\Phi\rangle_{0}= & |\Phi\rangle_{A} \otimes|\Phi\rangle_{A^{\prime}} \otimes|\Phi\rangle_{N V}, \\
|\Phi\rangle_{1}= & {\left[\alpha_{1} \alpha_{2}|R\rangle_{A}|R\rangle_{A^{\prime}}|+1\rangle-\beta_{1} \alpha_{2}|L\rangle_{A}|R\rangle_{A^{\prime}}|-1\rangle\right.} \\
& \left.-\alpha_{1} \beta_{2}|R\rangle_{A}|L\rangle_{A^{\prime}}|-1\rangle+\beta_{1} \beta_{2}|L\rangle_{A}|L\rangle_{A^{\prime}}|+1\rangle\right] \\
& \otimes\left|\Phi_{k}\right\rangle_{A} \otimes\left|\Phi_{k}\right\rangle_{A^{\prime}} .
\end{aligned}
$$

Second, one performs Hadamard operations on photons $A$ and $A^{\prime}$ with the half-wave plates $H_{p 1}$ and $H_{p 1^{\prime}}$. The state of the whole system is changed into

$$
\begin{aligned}
|\Phi\rangle_{2}= & \frac{1}{2}\left[\alpha_{1} \alpha_{2}(|R\rangle+|L\rangle)_{A}(|R\rangle+|L\rangle)_{A^{\prime}}|+1\rangle\right. \\
& -\beta_{1} \alpha_{2}(|R\rangle-|L\rangle)_{A}(|R\rangle+|L\rangle)_{A^{\prime}}|-1\rangle \\
& -\alpha_{1} \beta_{2}(|R\rangle+|L\rangle)_{A}(|R\rangle-|L\rangle)_{A^{\prime}}|-1\rangle \\
& \left.+\beta_{1} \beta_{2}(|R\rangle-|L\rangle)_{A}(|R\rangle-|L\rangle)_{A^{\prime}}|+1\rangle\right] \\
& \otimes\left|\Phi_{k}\right\rangle_{A} \otimes\left|\Phi_{k}\right\rangle_{A^{\prime}} .
\end{aligned}
$$

Third, one lets photon $A\left(A^{\prime}\right)$ pass though $C P B S_{5}$ $\left(C P B S_{5^{\prime}}\right), C P B S_{2}\left(C P B S_{2^{\prime}}\right)$, NV center, $C P B S_{3}$ $\left(C P B S_{3^{\prime}}\right), C P B S_{6}\left(C P B S_{6^{\prime}}\right)$, and $H_{p 2}\left(H_{p 2^{\prime}}\right)$, and the state of the whole system is changed from $|\Phi\rangle_{2}$ to $|\Phi\rangle_{3}$. Here

$$
\begin{aligned}
|\Phi\rangle_{3}= & {\left[\alpha_{1} \alpha_{2}|R\rangle_{A}|R\rangle_{A^{\prime}}|+1\rangle-\beta_{1} \alpha_{2}|R\rangle_{A}|L\rangle_{A^{\prime}}|-1\rangle\right.} \\
& \left.-\alpha_{1} \beta_{2}|L\rangle_{A}|R\rangle_{A^{\prime}}|-1\rangle+\beta_{1} \beta_{2}|L\rangle_{A}|L\rangle_{A^{\prime}}|+1\rangle\right] \\
& \otimes\left|\Phi_{k}\right\rangle_{A} \otimes\left|\Phi_{k}\right\rangle_{A^{\prime}} .
\end{aligned}
$$

Finally, by performing a Hadamard operation on the NV center, the state of the whole system is transformed into

$$
\begin{aligned}
|\Phi\rangle_{4}= & \frac{1}{\sqrt{2}}\left(\alpha_{2}|R\rangle-\beta_{2}|L\rangle\right)_{A}\left|\Phi_{k}\right\rangle_{A} \\
& \otimes\left(\alpha_{1}|R\rangle-\beta_{1}|L\rangle\right)_{A^{\prime}}\left|\Phi_{k}\right\rangle_{A^{\prime}} \otimes|+1\rangle \\
& +\frac{1}{\sqrt{2}}\left(\alpha_{2}|R\rangle+\beta_{2}|L\rangle\right)_{A}\left|\Phi_{k}\right\rangle_{A} \\
& \otimes\left(\alpha_{1}|R\rangle+\beta_{1}|L\rangle\right)_{A^{\prime}}\left|\Phi_{k}\right\rangle_{A^{\prime}} \otimes|-1\rangle .
\end{aligned}
$$

By measuring the NV center in the orthogonal basis $\{|+1\rangle,|-1\rangle\}$, the polarization state of photon $A$ is swaped with the polarization state of photon $A^{\prime}$ without disturbing their states in the spatial-mode DOFs. If the NV center is projected into state $|+1\rangle$, phase-flip operations $\sigma_{z}^{P}=|R\rangle\langle R|-| L\rangle\langle L|$ are performed on the polarization DOF of photons $A$ and $A^{\prime}$. After the phase-flip 
operations, the state of the two photons is transformed into

$$
\begin{aligned}
|\Phi\rangle_{f 1}= & \left(\alpha_{2}|R\rangle+\beta_{2}|L\rangle\right)_{A}\left|\Phi_{k}\right\rangle_{A} \\
& \otimes\left(\alpha_{1}|R\rangle+\beta_{1}|L\rangle\right)_{A^{\prime}}\left|\Phi_{k}\right\rangle_{A^{\prime}} .
\end{aligned}
$$

Here, $|\Phi\rangle_{f 1}$ is the objective state of the P-P-SWAP gate. If the NV center is projected into state $|-1\rangle$, the objective state can be obtained directly without phase-flip operations.

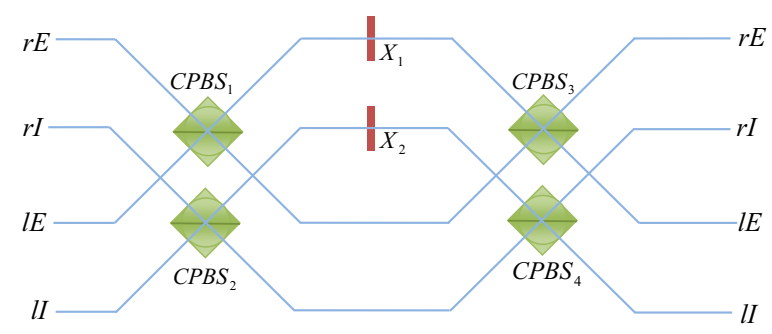

(a)
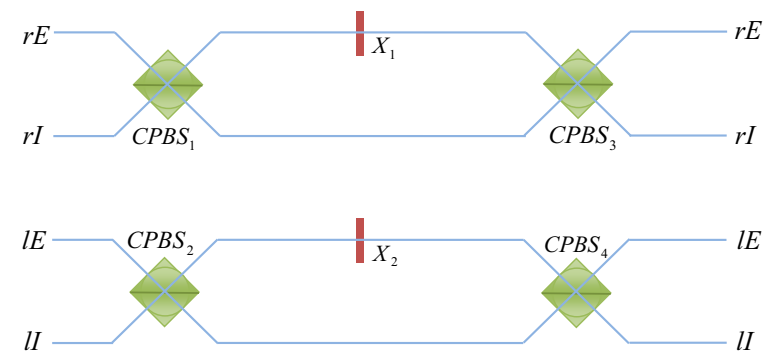

(b)

FIG. 6: (a) Schematic diagram of the SWAP gate between the polarization state and the spatial-mode state in the firstlongitudinal-momentum DOF of one photon. (b) Schematic diagram of the SWAP gate between the polarization state and the spatial-mode state in the second-longitudinal-momentum DOF of one photon. $X_{i}(i=1,2)$ is a half-wave plate which performs a polarization bit-flip operation $\sigma_{x}^{P}=|R\rangle\langle L|+$ $|L\rangle\langle R|$ on the photon.

\section{B. SWAP gate between the polarization state and the spatial state of one photon}

The schematic diagram of our SWAP gate between the polarization state and the spatial-mode state in the first-longitudinal-momentum DOF of one photon (P-FSWAP) is shown in Fig. 6(a), which is constructed with linear optical elements such as $C P B S_{i}(i=1,2,3,4)$ and half-wave plate $X_{i}(i=1,2)$. One can inject photon $A$, which is in the state described as Eq. (14), into the quantum circuit shown in Fig. 6(a). After photon $A$ passes through the quantum circuit shown in Fig. 6(a), the state of photon $A$ is transformed into

$$
\begin{aligned}
|\Phi\rangle_{f 2}= & \left(\gamma_{1}|R\rangle+\delta_{1}|L\rangle\right) \otimes\left(\alpha_{1}|r\rangle+\beta_{1}|l\rangle\right) \\
& \otimes\left(\varepsilon_{1}|E\rangle+\xi_{1}|I\rangle\right) .
\end{aligned}
$$

Here $|\Phi\rangle_{f 2}$ is the objective state of the P-F-SWAP gate. The schematic diagram of our SWAP gate between the polarization state and the spatial-mode state in the second longitudinal-momentum DOF of one photon (P-SSWAP gate) is shown in Fig. 6(b). After photon $A$, whose initial state is described as Eq. (14), passes through the circuit shown in Fig. 6(b), the state is changed into

$$
\begin{aligned}
|\Phi\rangle_{f 3}= & \left(\varepsilon_{1}|R\rangle+\xi_{1}|L\rangle\right) \otimes\left(\gamma_{1}|r\rangle+\delta_{1}|l\rangle\right) \\
& \otimes\left(\alpha_{1}|E\rangle+\beta_{1}|I\rangle\right) .
\end{aligned}
$$

Here, $|\Phi\rangle_{f 3}$ is just the objective state of the P-S-SWAP gate.

\section{EFFICIENT HYPER-EPP FOR MIXED TWO-PHOTON SIX-QUBIT HYPERENTANGLED BELL STATES}

In the practical transmission of photons in hyperentangled Bell states for high-capacity quantum communication, both the bit-flip error and the phase-flip error will occur on the photon systems. Although a phase-flip error cannot be directly purified, it can be transformed into a bit-flip error using a bilateral local operation 24 28. If a bit-flip error purification has been successfully solved, phase-flip errors also can be solved perfectly. In this way the two parties in quantum communication, say Alice and Bob, can purify a general mixed hyperentangled state. Below, we only discuss the purification of the two-photon six-qubit hyperentangled mixed state with bit-flip errors in the three DOFs.

Two identical two-photon six-qubit systems in mixed hyperentangled Bell states in the polarization DOF and two longitudinal-momentum DOFs with bit-flip errors can be described as follows:

$$
\begin{aligned}
\rho_{A B}= & {\left[F_{1}\left|\phi^{+}\right\rangle_{A B}^{p}\left\langle\phi^{+}\left|+\left(1-F_{1}\right)\right| \psi^{+}\right\rangle_{A B}^{p}\left\langle\psi^{+}\right|\right] } \\
& \otimes\left[F_{2}\left|\phi^{+}\right\rangle_{A B}^{F}\left\langle\phi^{+}\left|+\left(1-F_{2}\right)\right| \psi^{+}\right\rangle_{A B}^{F}\left\langle\psi^{+}\right|\right] \\
& \otimes\left[F_{3}\left|\phi^{+}\right\rangle_{A B}^{S}\left\langle\phi^{+}\left|+\left(1-F_{3}\right)\right| \psi^{+}\right\rangle_{A B}^{S}\left\langle\psi^{+}\right|\right], \\
\rho_{C D}= & {\left[F_{1}\left|\phi^{+}\right\rangle_{C D}^{p}\left\langle\phi^{+}\left|+\left(1-F_{1}\right)\right| \psi^{+}\right\rangle_{C D}^{p}\left\langle\psi^{+}\right|\right] } \\
& \otimes\left[F_{2}\left|\phi^{+}\right\rangle_{C D}^{F}\left\langle\phi^{+}\left|+\left(1-F_{2}\right)\right| \psi^{+}\right\rangle_{C D}^{F}\left\langle\psi^{+}\right|\right] \\
& \otimes\left[F_{3}\left|\phi^{+}\right\rangle_{C D}^{S}\left\langle\phi^{+}\left|+\left(1-F_{3}\right)\right| \psi^{+}\right\rangle_{C D}^{S}\left\langle\psi^{+}\right|\right] .
\end{aligned}
$$

Here the subscripts $A B$ and $C D$ represent two photon pairs shared by the two parties in quantum communication, say Alice and Bob. Alice holds the photons $A$ and $C$, and Bob holds the photons $B$ and $D . F_{1}, F_{2}$, and $F_{3}$ represent the probabilities of states $\left|\phi^{+}\right\rangle_{A B}^{p}\left(\left|\phi^{+}\right\rangle_{C D}^{p}\right)$, $\left|\phi^{+}\right\rangle_{A B}^{F}\left(\left|\phi^{+}\right\rangle_{C D}^{F}\right)$, and $\left|\phi^{+}\right\rangle_{A B}^{S}\left(\left|\phi^{+}\right\rangle_{C D}^{S}\right)$ in the mixed states, respectively.

The initial state of the system composed of the two identical two-photon six-qubit subsystems $A B C D$ can be expressed as $\rho_{0}=\rho_{A B} \otimes \rho_{C D}$. It can be viewed as a mixture of maximally hyperentangled pure states. In the polarization DOF, it is a mixture of the states 
$\left|\phi^{+}\right\rangle_{A B}^{p} \otimes\left|\phi^{+}\right\rangle_{C D}^{p},\left|\phi^{+}\right\rangle_{A B}^{p} \otimes\left|\psi^{+}\right\rangle_{C D}^{p},\left|\psi^{+}\right\rangle_{A B}^{p} \otimes\left|\phi^{+}\right\rangle_{C D}^{p}$, and $\left|\psi^{+}\right\rangle_{A B}^{p} \otimes\left|\psi^{+}\right\rangle_{C D}^{p}$ with the probabilities $F_{1}^{2}$, (1$\left.F_{1}\right) F_{1},\left(1-F_{1}\right) F_{1}$, and $\left(1-F_{1}\right)^{2}$, respectively. In the first-longitudinal-momentum DOF, it is a mixture of the states $\left|\phi^{+}\right\rangle_{A B}^{F} \otimes\left|\phi^{+}\right\rangle_{C D}^{F},\left|\phi^{+}\right\rangle_{A B}^{F} \otimes\left|\psi^{+}\right\rangle_{C D}^{F},\left|\psi^{+}\right\rangle_{A B}^{F_{A B}} \otimes$ $\left|\phi^{+}\right\rangle_{C D}^{F}$, and $\left|\psi^{+}\right\rangle_{A B}^{F} \otimes\left|\psi^{+}\right\rangle_{C D}^{F}$ with the probabilities $F_{2}^{2},\left(1-F_{2}\right) F_{2},\left(1-F_{2}\right) F_{2}$, and $\left(1-F_{2}\right)^{2}$, respectively. In the second-longitudinal-momentum DOF, it is a mixture of the states $\left|\phi^{+}\right\rangle_{A B}^{S} \otimes\left|\phi^{+}\right\rangle_{C D}^{S},\left|\phi^{+}\right\rangle_{A B}^{S} \otimes\left|\psi^{+}\right\rangle_{C D}^{S}$, $\left|\psi^{+}\right\rangle_{A B}^{S} \otimes\left|\phi^{+}\right\rangle_{C D}^{S}$, and $\left|\psi^{+}\right\rangle_{A B}^{S} \otimes\left|\psi^{+}\right\rangle_{C D}^{S}$ with the probabilities $F_{3}^{2},\left(1-F_{3}\right) F_{3},\left(1-F_{3}\right) F_{3}$, and $\left(1-F_{3}\right)^{2}$, respectively.

Our hyper-EPP for the nonlocal two-photon six-qubit systems in hyperentangled Bell states with bit-flip errors in the polarization DOF and the two longitudinalmomentum DOFs can be achieved with two steps in each round. The first step is completed with polarization and spatial-mode parity-check QNDs introduced in Sec. III. The second step of our hyper-EPP scheme is completed with the SWAP gates introduced in Sec. IV] We discuss the principles of these two steps in detail as follows.

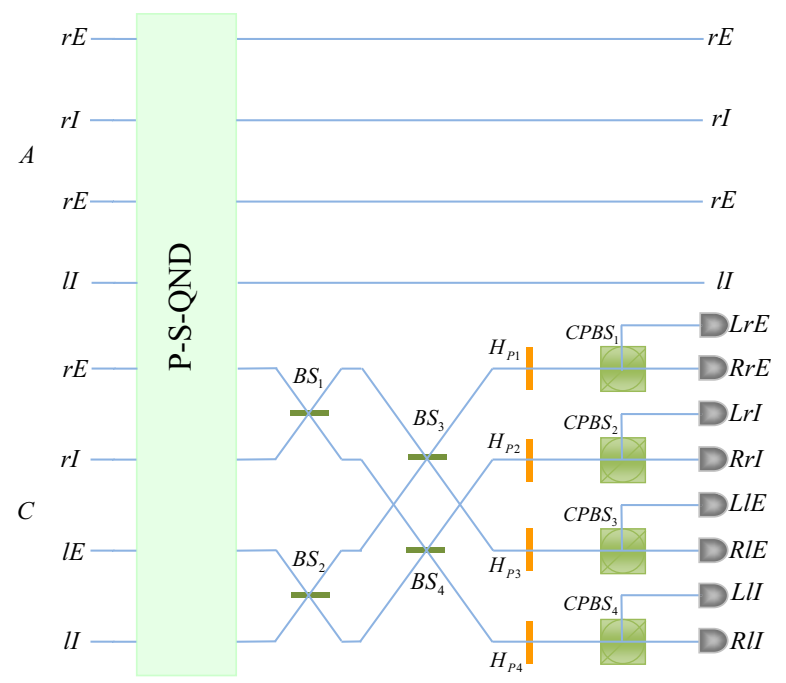

FIG. 7: Schematic diagram of the first step of our hyper-EPP for mixed hyperentangled Bell states with bit-flip errors in the polarization DOF and the two longitudinal-momentum DOFs with P-S-QNDs. A beam splitter $B S_{i}(i=1,2)$ is used to perform the Hadamard operation $\left[|E\rangle \rightarrow \frac{1}{\sqrt{2}}(|E\rangle+\right.$ $\left.|I\rangle),|I\rangle \rightarrow \frac{1}{\sqrt{2}}(|E\rangle-|I\rangle)\right]$ on the first-longitudinal-momentum DOF of a photon and $B S_{j}(j=3,4)$ is used to perform the Hadamard operation $\left[|r\rangle \rightarrow \frac{1}{\sqrt{2}}(|r\rangle+|l\rangle),|l\rangle \rightarrow \frac{1}{\sqrt{2}}(|r\rangle-|l\rangle)\right]$ on the second-longitudinal-momentum DOF of a photon. $D_{k}$ ( $k=\operatorname{Lr} E, \operatorname{Rr} E, L r I, \operatorname{Rr} I, L l E, R l E, L l I$, or RlI $)$ is a singlephoton detector.

\section{A. The first step of our hyper-EPP with} polarization and spatial-mode parity-check QND

The principle of the first step of our hyper-EPP is shown in Fig. 7]. Alice performs the P-S-QNDs on pho- tons $A C$ and performs the Hadamard operations on the polarization DOF and the spatial-mode DOFs of photon $C$. Bob performs the same operations on photons $B D$.

First, Alice and Bob perform the P-S-QNDs on the two two-photon systems $A C$ and $B D$, respectively. Based on the results of the P-S-QNDs on the identical two-photon systems, the states can be classified into eight cases. In case (1), the two identical twophoton systems $A C$ and $B D$ are in the same paritymode in all of the three DOFs, including the polarization DOF, the first-longitudinal-momentum DOF, and the second-longitudinal-momentum DOF. This case corresponds to the states $\left|\phi^{+}\right\rangle_{A B}^{p}\left|\phi^{+}\right\rangle_{C D}^{p}$ or $\left|\psi^{+}\right\rangle_{A_{B}}^{p}\left|\psi^{+}\right\rangle_{C D}^{p}$ in the polarization DOF, the states $\left|\phi^{+}\right\rangle_{A B}^{F}\left|\phi^{+}\right\rangle_{C D}^{F}$ or $\left|\psi^{+}\right\rangle_{A B}^{F}\left|\psi^{+}\right\rangle_{C D}^{F}$ in the first-longitudinal-momentum DOF, and the states $\left|\phi^{+}\right\rangle_{A B}^{S}\left|\phi^{+}\right\rangle_{C D}^{S}$ or $\left|\psi^{+}\right\rangle_{A B}^{S}\left|\psi^{+}\right\rangle_{C D}^{S}$ in the second-longitudinal-momentum DOF. The classification of the eight cases is shown in Table [.

Now, let us discuss case (1), in which the parity modes of $A C$ and $B D$ are same in the polarization DOF, the first-longitudinal-momentum DOF, and the secondlongitudinal-momentum DOF, to bring to light of the principle of the first step in our hyper-EPP. Case (2), in which the parity modes of $A C$ and $B D$ are different in the polarization DOF, the first-longitudinal-momentum DOF, and the second-longitudinal-momentum DOF, is discussed in the Appendix. The other cases are similar to these two cases with a little modification. When $A C$ and $B D$ are both in the even-parity modes in the polarization DOF, the first-longitudinal-momentum DOF, and the second longitudinal momentum DOF, Alice and Bob obtain the states

$$
\begin{aligned}
\left|\Phi_{1}\right\rangle^{p} & =\frac{1}{\sqrt{2}}(|R R R R\rangle+|L L L L\rangle)_{A B C D}, \\
\left|\Phi_{2}\right\rangle^{p} & =\frac{1}{\sqrt{2}}(|R L R L\rangle+|L R L R\rangle)_{A B C D}, \\
\left|\Phi_{1}\right\rangle^{F} & =\frac{1}{\sqrt{2}}(|r r r r\rangle+|l l l l\rangle)_{A B C D}, \\
\left|\Phi_{2}\right\rangle^{F} & =\frac{1}{\sqrt{2}}(|r l r l\rangle+|\operatorname{lrlr}\rangle)_{A B C D}, \\
\left|\Phi_{1}\right\rangle^{S} & =\frac{1}{\sqrt{2}}(|E E E E\rangle+|I I I I\rangle)_{A B C D}, \\
\left|\Phi_{2}\right\rangle^{S} & =\frac{1}{\sqrt{2}}(|E I E I\rangle+|I E I E\rangle)_{A B C D} .
\end{aligned}
$$

On the contrary, when $A C$ and $B D$ are both in the odd-parity modes in the polarization DOF, the first-longitudinal-momentum DOF, and the secondlongitudinal-momentum DOF, Alice and Bob obtain the 
TABLE I: The classification based on the results of the P-SQNDs on photons $A C$ and $B D$.

\begin{tabular}{|c|c|c|c|}
\hline Case & DOF & Parity-mode & Bell states \\
\hline (1) & $\begin{array}{l}\mathrm{p} \\
\mathrm{F} \\
\mathrm{S}\end{array}$ & $\begin{array}{l}\text { Same } \\
\text { Same } \\
\text { Same }\end{array}$ & $\begin{array}{l}\left|\phi^{+}\right\rangle_{A B}^{p}\left|\phi^{+}\right\rangle_{C D}^{p} \text { or }\left|\psi^{+}\right\rangle_{A B}^{p}\left|\psi^{+}\right\rangle_{C D}^{p} \\
\left|\phi^{+}\right\rangle_{A B}^{F}\left|\phi^{+}\right\rangle_{C D}^{F} \text { or }\left|\psi^{+}\right\rangle_{A B}^{F}\left|\psi^{+}\right\rangle_{C D}^{F} \\
\left|\phi^{+}\right\rangle_{A B}^{S}\left|\phi^{+}\right\rangle_{C D}^{S} \text { or }\left|\psi^{+}\right\rangle_{A B}^{S}\left|\psi^{+}\right\rangle_{C D}^{S}\end{array}$ \\
\hline$(2)$ & $\begin{array}{l}\mathrm{p} \\
\mathrm{F} \\
\mathrm{S}\end{array}$ & $\begin{array}{l}\text { different } \\
\text { Different } \\
\text { Different }\end{array}$ & $\begin{array}{l}\left|\phi^{+}\right\rangle_{A B}^{p}\left|\psi^{+}\right\rangle_{C D}^{p} \text { or }\left|\psi^{+}\right\rangle_{A B}^{p}\left|\phi^{+}\right\rangle_{C D}^{p} \\
\left|\phi^{+}\right\rangle_{A B}^{F}\left|\psi^{+}\right\rangle_{C D}^{F} \text { or }\left|\psi^{+}\right\rangle_{A B}^{F}\left|\phi^{+}\right\rangle_{C D}^{F} \\
\left|\phi^{+}\right\rangle_{A B}^{S}\left|\psi^{+}\right\rangle_{C D}^{S} \text { or }\left|\psi^{+}\right\rangle_{A B}^{S}\left|\phi^{+}\right\rangle_{C D}^{S}\end{array}$ \\
\hline (3) & $\begin{array}{l}\mathrm{p} \\
\mathrm{F} \\
\mathrm{S}\end{array}$ & $\begin{array}{l}\text { Different } \\
\text { Same } \\
\text { Same }\end{array}$ & $\begin{array}{l}\left|\phi^{+}\right\rangle_{A B}^{p}\left|\psi^{+}\right\rangle_{C D}^{p} \text { or }\left|\psi^{+}\right\rangle_{A B}^{p}\left|\phi^{+}\right\rangle_{C D}^{p} \\
\left|\phi^{+}\right\rangle_{A B}^{F}\left|\phi^{+}\right\rangle_{C D}^{F} \text { or }\left|\psi^{+}\right\rangle_{A B}^{F}\left|\psi^{+}\right\rangle_{C D}^{F} \\
\left|\phi^{+}\right\rangle_{A B}^{S}\left|\phi^{+}\right\rangle_{C D}^{S} \text { or }\left|\psi^{+}\right\rangle_{A B}^{S}\left|\psi^{+}\right\rangle_{C D}^{S}\end{array}$ \\
\hline (4) & $\begin{array}{l}\mathrm{p} \\
\mathrm{F} \\
\mathrm{S}\end{array}$ & $\begin{array}{l}\text { Same } \\
\text { Different } \\
\text { Same }\end{array}$ & $\begin{array}{l}\left|\phi^{+}\right\rangle_{A B}^{p}\left|\phi^{+}\right\rangle_{C D}^{p} \text { or }\left|\psi^{+}\right\rangle_{A B}^{p}\left|\psi^{+}\right\rangle_{C D}^{p} \\
\left|\phi^{+}\right\rangle_{A B}^{F}\left|\psi^{+}\right\rangle_{C D}^{F} \text { or }\left|\psi^{+}\right\rangle_{A B}^{F}\left|\phi^{+}\right\rangle_{C D}^{F} \\
\left|\phi^{+}\right\rangle_{A B}^{S}\left|\phi^{+}\right\rangle_{C D}^{S} \text { or }\left|\psi^{+}\right\rangle_{A B}^{S}\left|\psi^{+}\right\rangle_{C D}^{S}\end{array}$ \\
\hline$(5)$ & $\begin{array}{l}\mathrm{p} \\
\mathrm{F} \\
\mathrm{S}\end{array}$ & $\begin{array}{c}\text { Same } \\
\text { Same } \\
\text { Different }\end{array}$ & $\begin{array}{l}\left|\phi^{+}\right\rangle_{A B}^{p}\left|\phi^{+}\right\rangle_{C D}^{p} \text { or }\left|\psi^{+}\right\rangle_{A B}^{p}\left|\psi^{+}\right\rangle_{C D}^{p} \\
\left|\phi^{+}\right\rangle_{A B}^{F}\left|\phi^{+}\right\rangle_{C D}^{F} \text { or }\left|\psi^{+}\right\rangle_{A B}^{F}\left|\psi^{+}\right\rangle_{C D}^{F} \\
\left|\phi^{+}\right\rangle_{A B}^{S}\left|\psi^{+}\right\rangle_{C D}^{S} \text { or }\left|\psi^{+}\right\rangle_{A B}^{S}\left|\phi^{+}\right\rangle_{C D}^{S}\end{array}$ \\
\hline$(6)$ & $\begin{array}{l}\mathrm{p} \\
\mathrm{F} \\
\mathrm{S}\end{array}$ & $\begin{array}{l}\text { Different } \\
\text { Different } \\
\text { Same }\end{array}$ & $\begin{array}{l}\left|\phi^{+}\right\rangle_{A B}^{p}\left|\psi^{+}\right\rangle_{C D}^{p} \text { or }\left|\psi^{+}\right\rangle_{A B}^{p}\left|\phi^{+}\right\rangle_{C D}^{p} \\
\left|\phi^{+}\right\rangle_{A B}^{F}\left|\psi^{+}\right\rangle_{C D}^{F} \text { or }\left|\psi^{+}\right\rangle_{A B}^{F}\left|\phi^{+}\right\rangle_{C D}^{F} \\
\left|\phi^{+}\right\rangle_{A B}^{S}\left|\phi^{+}\right\rangle_{C D}^{S} \text { or }\left|\psi^{+}\right\rangle_{A B}^{S}\left|\psi^{+}\right\rangle_{C D}^{S}\end{array}$ \\
\hline (7) & $\begin{array}{l}\mathrm{p} \\
\mathrm{F} \\
\mathrm{S}\end{array}$ & $\begin{array}{l}\text { Different } \\
\text { Same } \\
\text { Different }\end{array}$ & $\begin{array}{l}\left|\phi^{+}\right\rangle_{A B}^{p}\left|\psi^{+}\right\rangle_{C D}^{p} \text { or }\left|\psi^{+}\right\rangle_{A B}^{p}\left|\phi^{+}\right\rangle_{C D}^{p} \\
\left|\phi^{+}\right\rangle_{A B}^{F}\left|\phi^{+}\right\rangle_{C D}^{F} \text { or }\left|\psi^{+}\right\rangle_{A B}^{F}\left|\psi^{+}\right\rangle_{C D}^{F} \\
\left|\phi^{+}\right\rangle_{A B}^{S}\left|\psi^{+}\right\rangle_{C D}^{S} \text { or }\left|\psi^{+}\right\rangle_{A B}^{S}\left|\phi^{+}\right\rangle_{C D}^{S}\end{array}$ \\
\hline$(8)$ & $\begin{array}{l}\mathrm{p} \\
\mathrm{F} \\
\mathrm{S}\end{array}$ & $\begin{array}{c}\text { Same } \\
\text { Different } \\
\text { Different }\end{array}$ & $\begin{array}{l}\left|\phi^{+}\right\rangle_{A B}^{p}\left|\phi^{+}\right\rangle_{C D}^{p} \text { or }\left|\psi^{+}\right\rangle_{A B}^{p}\left|\psi^{+}\right\rangle_{C D}^{p} \\
\left|\phi^{+}\right\rangle_{A B}^{F}\left|\psi^{+}\right\rangle_{C D}^{F} \text { or }\left|\psi^{+}\right\rangle_{A B}^{F}\left|\phi^{+}\right\rangle_{C D}^{F} \\
\left|\phi^{+}\right\rangle_{A B}^{S}\left|\psi^{+}\right\rangle_{C D}^{S} \text { or }\left|\psi^{+}\right\rangle_{A B}^{S}\left|\phi^{+}\right\rangle_{C D}^{S}\end{array}$ \\
\hline
\end{tabular}

states

$$
\begin{aligned}
\left|\Phi_{3}\right\rangle^{p} & =\frac{1}{\sqrt{2}}(|R R L L\rangle+|L L R R\rangle)_{A B C D}, \\
\left|\Phi_{4}\right\rangle^{p} & =\frac{1}{\sqrt{2}}(|R L L R\rangle+|L R R L\rangle)_{A B C D}, \\
\left|\Phi_{3}\right\rangle^{F} & =\frac{1}{\sqrt{2}}(|r r l l\rangle+|l l r r\rangle)_{A B C D}, \\
\left|\Phi_{4}\right\rangle^{F} & =\frac{1}{\sqrt{2}}(|r l l r\rangle+|l r r l\rangle)_{A B C D}, \\
\left|\Phi_{3}\right\rangle^{S} & =\frac{1}{\sqrt{2}}(|E E I I\rangle+|I I E E\rangle)_{A B C D}, \\
\left|\Phi_{4}\right\rangle^{S} & =\frac{1}{\sqrt{2}}(|E I I E\rangle+|I E E I\rangle)_{A B C D} .
\end{aligned}
$$

The states $\left|\Phi_{3}\right\rangle^{p}$ and $\left|\Phi_{4}\right\rangle^{p}$ can be transformed into $\left|\Phi_{1}\right\rangle^{p}$ and $\left|\Phi_{2}\right\rangle^{p}$ by performing the bit-flip operations on pho- tons $C D$ in the polarization $\mathrm{DOF}$, respectively. Similarly, $\left|\Phi_{3}\right\rangle^{F}$ and $\left|\Phi_{4}\right\rangle^{F}$ can be transformed into $\left|\Phi_{1}\right\rangle^{F}$ and $\left|\Phi_{2}\right\rangle^{F}$ by the bit-flip operations on photons $C D$ in the first-longitudinal-momentum DOF, respectively. $\left|\Phi_{3}\right\rangle^{S}$ and $\left|\Phi_{4}\right\rangle^{S}$ can also be transformed into $\left|\Phi_{1}\right\rangle^{S}$ and $\left|\Phi_{2}\right\rangle^{S}$ by the bit-flip operations on photons $C D$ in the secondlongitudinal-momentum DOF, respectively.

Next, Alice and Bob perform Hadamard operations on photons $C$ and $D$ in the polarization and the two longitudinal-momentum DOFs, respectively. In the polarization DOF, the states $\left|\Phi_{1}\right\rangle^{p}$ and $\left|\Phi_{2}\right\rangle^{p}$ are transformed into the states $\left|\Phi_{1^{\prime}}\right\rangle^{p}$ and $\left|\Phi_{2^{\prime}}\right\rangle^{p}$, respectively. In the first-longitudinal-momentum DOF, the states $\left|\Phi_{1}\right\rangle^{F}$ and $\left|\Phi_{2}\right\rangle^{F}$ are transformed into $\left|\Phi_{1}^{\prime}\right\rangle^{F}$ and $\left|\Phi_{2}^{\prime}\right\rangle^{F}$, respectively. In the second-longitudinal-momentum DOF, the states $\left|\Phi_{1}\right\rangle^{S}$ and $\left|\Phi_{2}\right\rangle^{S}$ are transformed into $\left|\Phi_{1}^{\prime}\right\rangle^{S}$ and $\left|\Phi_{2}^{\prime}\right\rangle^{S}$, respectively. Here

$$
\begin{aligned}
\left|\Phi_{1}^{\prime}\right\rangle^{p}= & \frac{1}{2 \sqrt{2}}\left[(|R R\rangle+|L L\rangle)_{A B}(|R R\rangle+|L L\rangle)_{C D}\right. \\
& \left.+(|R R\rangle-|L L\rangle)_{A B}(|R L\rangle+|L R\rangle)_{C D}\right], \\
\left|\Phi_{2}^{\prime}\right\rangle^{p}= & \frac{1}{2 \sqrt{2}}\left[(|R L\rangle+|L R\rangle)_{A B}(|R R\rangle-|L L\rangle)_{C D}\right. \\
& \left.+(-|R L\rangle+|L R\rangle)_{A B}(|R L\rangle-|L R\rangle)_{C D}\right], \\
\left|\Phi_{1}^{\prime}\right\rangle^{F}= & \frac{1}{2 \sqrt{2}}\left[(|r r\rangle+|l l\rangle)_{A B}(|r r\rangle+|l l\rangle)_{C D}\right. \\
& \left.+(|r r\rangle-|l l\rangle)_{A B}(|r l\rangle+|l r\rangle)_{C D}\right], \\
\left|\Phi_{2}^{\prime}\right\rangle^{F}= & \frac{1}{2 \sqrt{2}}\left[(|r l\rangle+|l r\rangle)_{A B}(|r r\rangle-|l l\rangle)_{C D}\right. \\
& \left.+(-|r l\rangle+|l r\rangle)_{A B}(|r l\rangle-|l r\rangle)_{C D}\right], \\
\left|\Phi_{1}^{\prime}\right\rangle^{S}= & \frac{1}{2 \sqrt{2}}\left[(|E E\rangle+|I I\rangle)_{A B}(|E E\rangle+|I I\rangle)_{C D}\right. \\
& \left.+(|E E\rangle-|I I\rangle)_{A B}(|E I\rangle+|I E\rangle)_{C D}\right], \\
\left|\Phi_{2}^{\prime}\right\rangle^{S}= & \frac{1}{2 \sqrt{2}}\left[(|E I\rangle+|I E\rangle)_{A B}(|E E\rangle-|I I\rangle)_{C D}\right. \\
& \left.+(-|E I\rangle+|I E\rangle)_{A B}(|E I\rangle-|I E\rangle)_{C D}\right] .
\end{aligned}
$$

Finally, the photons $C$ and $D$ are detected by singlephoton detectors, respectively. If the photons $C D$ are in the even-parity mode in the polarization DOF (the first-longitudinal-momentum DOF or the secondlongitudinal-momentum DOF), nothing is needed to be done. If the photons $C D$ are in the odd-parity mode in the polarization DOF (the first-longitudinal-momentum DOF or the second-longitudinal-momentum DOF), the phase-flip operation $\sigma_{z}^{p}=|R\rangle\langle R|-| L\rangle\langle L|\left(\sigma_{z}^{F}=|r\rangle\langle r|-\right.$ $|l\rangle\langle l|$ or $\left.\sigma_{z}^{S}=|E\rangle\langle E|-| I\rangle\langle I|\right)$ is operated on the photon $B$.

After the first step of our hyper-EPP, Alice and Bob obtain the states $\left|\phi^{+}\right\rangle^{p(F, S)}$ and $\left|\psi^{+}\right\rangle^{p(F, S)}$ with different probabilities corresponding to the eight cases, respectively, which are shown in Table II If the two two-photon systems $A B C D$ are projected into the states in case (1), the hyper-EPP of the system $A B$ is completed. If the two two-photon systems $A B C D$ are projected into case 
TABLE II: The probabilities of different states corresponding to the eight cases, respectively.

\begin{tabular}{ccccccc}
\hline \hline \multirow{2}{*}{ Case } & \multicolumn{5}{c}{ Probability } \\
\cline { 2 - 6 } & $\left|\phi^{+}\right\rangle_{A B}^{p}$ & $\left|\psi^{+}\right\rangle_{A B}^{p}$ & $\left|\phi^{+}\right\rangle_{A B}^{F}$ & $\left|\psi^{+}\right\rangle_{A B}^{F}$ & $\left|\phi^{+}\right\rangle_{A B}^{S}$ & $\left|\psi^{+}\right\rangle_{A B}^{S}$ \\
\hline$(1)$ & $F_{1}^{2}$ & $\left(1-F_{1}\right)^{2}$ & $F_{2}^{2}$ & $\left(1-F_{2}\right)^{2}$ & $F_{3}^{2}$ & $\left(1-F_{3}\right)^{2}$ \\
$(2)$ & $F_{1}\left(1-F_{1}\right)$ & $F_{1}\left(1-F_{1}\right)$ & $F_{2}\left(1-F_{2}\right)$ & $F_{2}\left(1-F_{2}\right)$ & $F_{3}\left(1-F_{3}\right)$ & $F_{3}\left(1-F_{3}\right)$ \\
$(3)$ & $F_{1}\left(1-F_{1}\right)$ & $F_{1}\left(1-F_{1}\right)$ & $F_{2}^{2}$ & $\left(1-F_{2}\right)^{2}$ & $\left(1-F_{3}\right)^{2}$ & $\left(1-F_{3}\right)^{2}$ \\
$(4)$ & $F_{1}^{2}$ & $\left(1-F_{1}\right)^{2}$ & $F_{2}\left(1-F_{2}\right)$ & $F_{2}\left(1-F_{2}\right)$ & $F_{3}^{2}$ & $\left(1-F_{3}\right)^{2}$ \\
$(5)$ & $F_{1}^{2}$ & $\left(1-F_{1}\right)^{2}$ & $F_{2}^{2}$ & $\left(1-F_{2}\right)^{2}$ & $F_{3}\left(1-F_{3}\right)$ & $F_{3}\left(1-F_{3}\right)$ \\
$(6)$ & $F_{1}\left(1-F_{1}\right)$ & $F_{1}\left(1-F_{1}\right)$ & $F_{2}\left(1-F_{2}\right)$ & $F_{2}\left(1-F_{2}\right)$ & $F_{3}^{2}$ & $\left(1-F_{3}\right)^{2}$ \\
$(7)$ & $F_{1}\left(1-F_{1}\right)$ & $F_{1}\left(1-F_{1}\right)$ & $F_{2}^{2}$ & $\left(1-F_{2}\right)^{2}$ & $F_{3}\left(1-F_{3}\right)$ & $F_{3}\left(1-F_{3}\right)$ \\
$(8)$ & $F_{1}^{2}$ & $\left(1-F_{1}\right)^{2}$ & $F_{2}\left(1-F_{2}\right)$ & $F_{2}\left(1-F_{2}\right)$ & $F_{3}\left(1-F_{3}\right)$ & $F_{3}\left(1-F_{3}\right)$ \\
\hline \hline
\end{tabular}

(2), Alice and Bob will discard the two photons. Otherwise, the second step of hyper-EPP with SWAP gates is required if the two two-photon systems $A B C D$ are projected into case $(i)(i=3 \sim 8)$.

\section{B. The second step of our hyper-EPP with SWAP gates}

If the two two-photon systems $A B$ and $C D$ are projected into the case (6), the fidelities of the Bell states of the photon pair $A B$ in the polarization and the firstlongitudinal-momentum DOFs are lower than the initial ones and the fidelity in the second-longitudinalmomentum DOF is higher than the initial one. Alice and Bob seek another two two-photon systems $A^{\prime} B^{\prime}$ and $C^{\prime} D^{\prime}$ projected into the case (5), whose fidelity is lower than the initial one in the second-longitudinalmomentum DOF and higher than the initial one in the polarization and the first-longitudinal-momentum DOFs, respectively. The photons $A, C, A^{\prime}$, and $C^{\prime}$ belong to $\mathrm{Al}-$ ice and $B, D, B^{\prime}$, and $D^{\prime}$ belong to Bob. We discuss the result of the second step when the states of the photons $A B$ and $A^{\prime} B^{\prime}$ are in $|\Phi\rangle_{A B 0}=\left|\psi^{+}\right\rangle^{p}\left|\phi^{+}\right\rangle^{F}\left|\phi^{+}\right\rangle^{S}$ and $|\Phi\rangle_{A^{\prime} B^{\prime} 0}=\left|\phi^{+}\right\rangle^{p}\left|\psi^{+}\right\rangle^{F}\left|\psi^{+}\right\rangle^{S}$, respectively, as an example.

First, Alice and Bob swap the polarization states of the two-photon system $A B$ and the states of the system $A^{\prime} B^{\prime}$ by using the P-P-SWAP gate shown in Fig. 5. The state of the system composed of fourphoton $A B A^{\prime} B^{\prime}$ and two NV centers are transformed from $|\Phi\rangle_{0}=|\Phi\rangle_{A B 0}|\Phi\rangle_{A^{\prime} B^{\prime} 0}\left|\phi_{+}\right\rangle_{a}\left|\phi_{+}\right\rangle_{b}$ to the state

$$
\begin{aligned}
|\Phi\rangle= & \frac{1}{4}[-(|R R R L\rangle+|L L R L\rangle \\
& +|R R L R\rangle+|L L L R\rangle)|+1\rangle_{a}|+1\rangle_{b} \\
& +(|R R R L\rangle-|L L R L\rangle \\
& -|R R L R\rangle+|L L L R\rangle)|+1\rangle_{a}|-1\rangle_{b} \\
& -(|R R R L\rangle-|L L R L\rangle \\
& -|R R L R\rangle+|L L L R\rangle)|-1\rangle_{a}|+1\rangle_{b} \\
& +(|R R R L\rangle+|L L R L\rangle \\
& \left.+|R R L R\rangle+|L L L R\rangle)|-1\rangle_{a}|-1\rangle_{b}\right] \\
& \otimes\left|\phi^{+}\right\rangle_{A B}^{F}\left|\phi^{+}\right\rangle_{A B}^{S}\left|\psi^{+}\right\rangle_{A^{\prime} B^{\prime}}^{F}\left|\psi^{+}\right\rangle_{A^{\prime} B^{\prime}}^{S}
\end{aligned}
$$

If the state of two NV centers $a$ and $b$ belonging to Alice and Bob is $|+1\rangle_{a}|+1\rangle_{b}$ or $|-1\rangle_{a}|-1\rangle_{b}$, Alice and Bob do nothing. If the state of the NV centers is $|+1\rangle_{a}|-1\rangle_{b}$ or $\mid-$ $1\rangle_{a}|+1\rangle_{b}$, Alice and Bob perform a $\sigma_{z}^{P}=|R\rangle\langle R|-| L\rangle\langle L|$ operation on photons $A^{\prime}$ and $B$, respectively. Thus, Alice and Bob obtain the states

$$
\begin{aligned}
& |\Phi\rangle_{A B 1}=\left|\phi^{+}\right\rangle^{p}\left|\phi^{+}\right\rangle^{F}\left|\phi^{+}\right\rangle^{S}, \\
& |\Phi\rangle_{A^{\prime} B^{\prime} 1}=\left|\psi^{+}\right\rangle^{p}\left|\psi^{+}\right\rangle^{F}\left|\psi^{+}\right\rangle^{S} .
\end{aligned}
$$

Second, Alice and Bob swap the polarization states and the first-longitudinal-momentum states of photons $A, B, A^{\prime}$, and $B^{\prime}$, respectively. The states of the two two-photon systems are transformed into the states

$$
\begin{aligned}
& |\Phi\rangle_{A B 2}=\left|\phi^{+}\right\rangle^{p}\left|\phi^{+}\right\rangle^{F}\left|\phi^{+}\right\rangle^{S}, \\
& |\Phi\rangle_{A^{\prime} B^{\prime} 2}=\left|\psi^{+}\right\rangle^{p}\left|\psi^{+}\right\rangle^{F}\left|\psi^{+}\right\rangle^{S} .
\end{aligned}
$$

Third, Alice and Bob swap the polarization states of the two systems $A B$ and $A^{\prime} B^{\prime}$ again and obtain the states

$$
\begin{aligned}
& |\Phi\rangle_{A B 3}=\left|\psi^{+}\right\rangle^{p}\left|\phi^{+}\right\rangle^{F}\left|\phi^{+}\right\rangle^{S}, \\
& |\Phi\rangle_{A^{\prime} B^{\prime} 3}=\left|\phi^{+}\right\rangle^{p}\left|\psi^{+}\right\rangle^{F}\left|\psi^{+}\right\rangle^{S} .
\end{aligned}
$$

Finally, Alice and Bob swap the polarization states and the first-longitudinal-momentum states of photons $A, B$, $A^{\prime}$, and $B^{\prime}$, respectively. The states of the two systems are changed into

$$
\begin{aligned}
& |\Phi\rangle_{A B 4}=\left|\phi^{+}\right\rangle^{p}\left|\psi^{+}\right\rangle^{F}\left|\phi^{+}\right\rangle^{S} \\
& |\Phi\rangle_{A^{\prime} B^{\prime} 4}=\left|\psi^{+}\right\rangle^{p}\left|\phi^{+}\right\rangle^{F}\left|\psi^{+}\right\rangle^{S} .
\end{aligned}
$$


Here $|\Phi\rangle_{A B 4}$ and $|\Phi\rangle_{A^{\prime} B^{\prime} 4}$ are the final states of this case in the second step of hyper-EPP.

If the two two-photon systems $A B$ and $C D$ are projected into the states in case (3), (4), (5), (7), or (8), the second step of the hyper-EPP can be completed, as discussed in the Appendix.
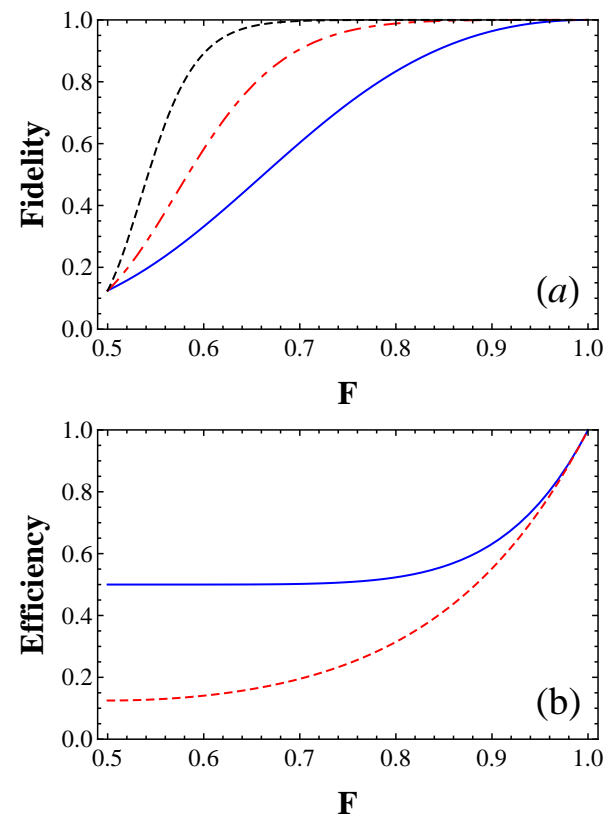

FIG. 8: (a) The fidelities of our hyper-EPP for mixed hyperentangled Bell states in three DOFs. The solid line, dashdotted line, and dashed line represent the fidelities of the iteration times $n=1,2$, and 3 , respectively, for the cases with $F_{1}=F_{2}=F_{3}=F$. (b) The efficiencies of the of our hyperEPP for mixed hyperentangled Bell states in three DOFs. The dashed line and the solid line represent the efficiencies of the first step and the two steps in the first round, respectively for the cases with $F_{1}=F_{2}=F_{3}=F$.

After these two steps, the first round of our hyperEPP process is completed and the state of the two-photon system $A B$ is changed to be

$$
\begin{aligned}
\rho_{A B}^{\prime}= & {\left[F_{1}^{\prime}\left|\phi^{+}\right\rangle_{A B}^{p}\left\langle\phi^{+}\left|+\left(1-F_{1}^{\prime}\right)\right| \psi^{+}\right\rangle_{A B}^{p}\left\langle\psi^{+}\right|\right] } \\
& \otimes\left[F_{2}^{\prime}\left|\phi^{+}\right\rangle_{A B}^{F}\left\langle\phi^{+}\left|+\left(1-F_{2}^{\prime}\right)\right| \psi^{+}\right\rangle_{A B}^{F}\left\langle\psi^{+}\right|\right] \\
& \otimes\left[F_{3}^{\prime}\left|\phi^{+}\right\rangle_{A B}^{S}\left\langle\phi^{+}\left|+\left(1-F_{3}^{\prime}\right)\right| \psi^{+}\right\rangle_{A B}^{S}\left\langle\psi^{+}\right|\right] .
\end{aligned}
$$

Here $F_{1}^{\prime}=\frac{F_{1}^{2}}{F_{1}^{2}+\left(1-F_{1}\right)^{2}}, \quad F_{2}^{\prime}=\frac{F_{2}^{2}}{F_{2}^{2}+\left(1-F_{2}\right)^{2}}$, and $F_{3}^{\prime}=$ $\frac{F_{3}^{2}}{F_{3}^{2}+\left(1-F_{3}\right)^{2}}$. The fidelity of the finale state is $F^{\prime}=$ $F_{1}^{\prime} F_{2}^{\prime} F_{3}^{\prime}$ which is higher than the initial fidelity $F=$ $F_{1} F_{2} F_{3}$ when $F_{i}>1 / 2(i=1,2,3)$. By iterating our hyper-EPP process, the fidelity of the state of the twophoton system can be improved dramatically. The fidelities of the final states are shown in Fig. 8(a) with iterating $n=1,2,3$ times for the case with $F_{1}=F_{2}=F_{3}=F$.

The efficiency of an EPP is defined as the probability of obtaining a high-fidelity entangled photon system from a pair of photon systems transmitted over a noisy channel without photon loss. The efficiency of our hyper-EPP after the first step in the first round is

$$
Y_{1}=\left[F_{1}^{2}+\left(1-F_{1}\right)^{2}\right]\left[F_{2}^{2}+\left(1-F_{2}\right)^{2}\right]\left[F_{3}^{2}+\left(1-F_{3}\right)^{2}\right] \text {. }
$$

After the second step, the efficiency of the first round of the hyper-EPP process is

$$
\begin{aligned}
& Y_{2}=[\left.F_{1}^{2}+\left(1-F_{1}\right)^{2}\right]\left[F_{2}^{2}+\left(1-F_{2}\right)^{2}\right]\left[F_{3}^{2}+\left(1-F_{3}\right)^{2}\right] \\
&+\min \left\{\left(\left[2 F_{1}\left(1-F_{1}\right)\right]\left[F_{2}^{2}+\left(1-F_{2}\right)^{2}\right]\right.\right. \\
& {\left.\left[F_{3}^{2}+\left(1-F_{3}\right)^{2}\right]\right),\left(\left[F_{1}^{2}+\left(1-F_{1}\right)^{2}\right]\right.} \\
& {\left.\left.\left[2 F_{2}\left(1-F_{2}\right)\right]\left[2 F_{3}\left(1-F_{3}\right)\right]\right)\right\} } \\
&+\min \left\{\left(\left[F_{1}^{2}+\left(1-F_{1}\right)^{2}\right]\left[2 F_{2}\left(1-F_{2}\right)\right]\right.\right. \\
& {\left.\left[F_{3}^{2}+\left(1-F_{3}\right)^{2}\right]\right),\left(\left[2 F_{1}\left(1-F_{1}\right)\right]\right.} \\
& {\left.\left.\left[F_{2}^{2}+\left(1-F_{2}\right)^{2}\right]\left[2 F_{3}\left(1-F_{3}\right)\right]\right)\right\} } \\
&+\min \left\{\left(\left[F_{1}^{2}+\left(1-F_{1}\right)^{2}\right]\left[F_{2}^{2}+\left(1-F_{2}\right)^{2}\right]\right.\right. \\
& {\left.\left[2 F_{3}\left(1-F_{3}\right)\right]\right),\left(\left[2 F_{1}\left(1-F_{1}\right)\right]\right.} \\
& {\left.\left.\left[2 F_{2}\left(1-F_{2}\right)\right]\left[F_{3}^{2}+\left(1-F_{3}\right)^{2}\right]\right)\right\} . }
\end{aligned}
$$

The efficiencies $Y_{1}$ and $Y_{2}$ of our hyper-EPP for the case with $F_{1}=F_{2}=F_{3}=F$ are shown in Fig. 8 (b), respectively. Obviously, the efficiency of our hyper-EPP is improved largely with the second step for purification.

\section{EXPANSION}

We present a hyper-EPP for the nonlocal twophoton systems in the polarization and two longitudinalmomentum DOFs by two steps. The first step is completed by the P-S-QNDs, Hadamard operations, and some detections. After the first step, we can obtain the information of the probability of each Bell state in different DOFs. If the probabilities of the three even-parity Bell states in the three DOFs are higher than the initial ones [corresponding to the case (1) in the first step], the second step is not need. If the probabilities of the even-parity Bell states in the three DOFs are all lower than the initial ones [corresponding to the case (2) in the first step], the two-photon system is discarded. Otherwise [corresponding to the case $(3),(4),(5),(6),(7)$ or (8)], the second step is required. The second step is completed by using the combination of the P-P-SWAP gate, the P-F-SWAP gate, and the P-S-SWAP gate. The purpose of the second step is to improve the probability of the even-parity Bell state in one or two DOFs by costing some other two-photon systems. For example, if the two two-photon systems $A B C D$ are projected into the states in case (4), the probability of the even-parity Bell state in the first-longitudinal-momentum DOF is lower than the initial one. We seek another two two-photon systems $A^{\prime} B^{\prime} C^{\prime} D^{\prime}$ whose probability of even-parity Bell states in the first-longitudinal-momentum DOF is higher than the initial one and the fidelities of the Bell states in another 
two DOFs are lower than the initial ones. Thus, with the P-P-SWAP gate and the P-F-SWAP gate, the fidelity of the two-photon system $A B$ is improved. If we can construct the SWAP gate between the polarization state and arbitrary another longitudinal-momentum state without affecting the states in other DOFs, the second step can be used to purify the two-photon systems in the polarization DOF and multiple longitudinal-momentum DOFs. That is to say, using SWAP gates is a universal method for purifying the two-photon systems in the polarization DOF and multiple longitudinal-momentum DOFs.

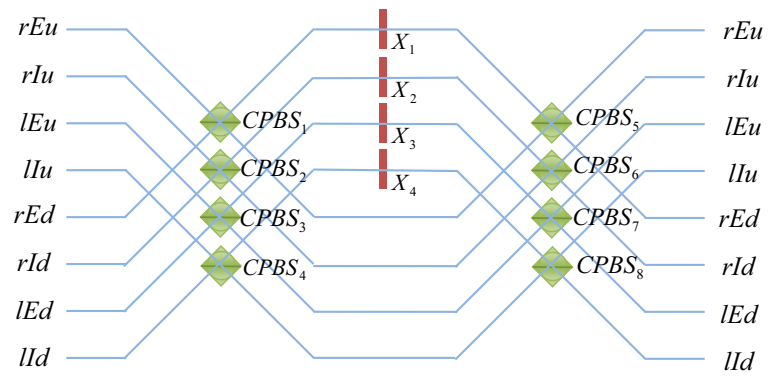

FIG. 9: Schematic diagram of the SWAP gate between the polarization state and the spatial-mode state in the third longitudinal-momentum DOF of one photon.

We construct a SWAP gate between the polarization state and the state in the third-longitudinal-momentum DOF of a photon (P-T-SWAP gate) of being simultaneously entangled in the polarization DOF and three longitudinal-momentum DOFs as an example. The initial state of a photon being simultaneously entangled with other photons in the polarization DOF and three longitudinal-momentum DOFs can be expressed as

$$
\begin{aligned}
|\Phi\rangle= & \left(a_{1}|R\rangle+b_{1}|L\rangle\right) \otimes\left(a_{2}|r\rangle+b_{2}|l\rangle\right) \\
& \otimes\left(a_{3}|E\rangle+b_{3}|I\rangle\right) \otimes\left(a_{4}|u\rangle+b_{4}|d\rangle\right) .
\end{aligned}
$$

Here $\left(a_{4}|u\rangle+b_{4}|d\rangle\right)$ is the state of the photon $A$ being entangled with other photons in the third-longitudinalmomentum DOF $\left(a_{4}\right.$ and $b_{4}$ include not only the parameters for photon $A$ but also the states for other photons, so do $a_{i}$ and $b_{i}$ ). $u$ and $d$ represent the upper and the down spatial-mode states of the photon, respectively. The schematic diagram of the P-T-SWAP gate, which is constructed with some linear optical elements, is shown in Fig. 9, After the photon $A$ passes through the quantum circuit, its state is transformed into

$$
\begin{aligned}
\left|\Phi^{\prime}\right\rangle= & \left(a_{4}|R\rangle+b_{4}|L\rangle\right) \otimes\left(a_{2}|r\rangle+b_{2}|l\rangle\right) \\
& \otimes\left(a_{3}|E\rangle+b_{3}|I\rangle\right) \otimes\left(a_{1}|u\rangle+b_{1}|d\rangle\right) .
\end{aligned}
$$

Here $\left|\Phi^{\prime}\right\rangle$ is the objective state of the P-T-SWAP gate. After the first step of the hyper-EPP, if the probability of the even-parity Bell state of the system $A B C D$ in the third longitudinal-momentum DOF is lower than the initial one, we can seek another system $A^{\prime} B^{\prime} C^{\prime} D^{\prime}$ whose probability of the even-parity Bell state in this
DOF is higher. By using the P-P-SWAP gate and the $\mathrm{P}$ T-SWAP gate, the second step of our hyper-EPP can be completed to improve the efficiency of purification largely.
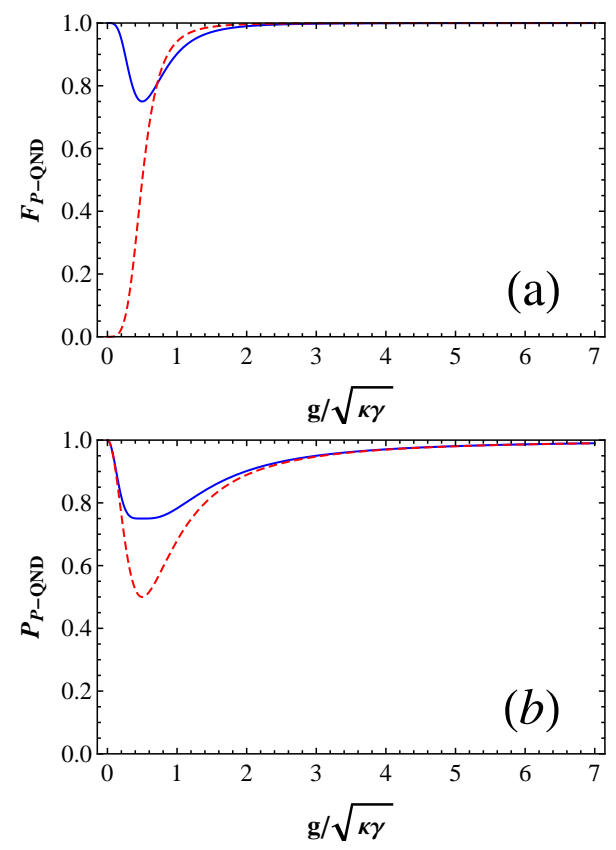

FIG. 10: (a) The fidelity of the polarization-parity check QND. (b) The efficiency of the polarization-parity check QND. The solid line represents $F_{P 1}\left(\eta_{P 1}\right)$ for $\left|\phi^{ \pm}\right\rangle^{p}\left|\phi^{ \pm}\right\rangle^{F}\left|\phi^{ \pm}\right\rangle^{S},\left|\phi^{ \pm}\right\rangle^{p}\left|\phi^{ \pm}\right\rangle^{F}\left|\psi^{ \pm}\right\rangle^{S},\left|\phi^{ \pm}\right\rangle^{p}\left|\psi^{ \pm}\right\rangle^{F}\left|\phi^{ \pm}\right\rangle^{S}$, and $\left|\phi^{ \pm}\right\rangle^{p}\left|\psi^{ \pm}\right\rangle^{F}\left|\psi^{ \pm}\right\rangle^{S}$. The dashed line represents $F_{P 2}\left(\eta_{P 2}\right)$ for $\left|\psi^{ \pm}\right\rangle^{p}\left|\phi^{ \pm}\right\rangle^{F}\left|\phi^{ \pm}\right\rangle^{S},\left|\psi^{ \pm}\right\rangle^{p}\left|\phi^{ \pm}\right\rangle^{F}\left|\psi^{ \pm}\right\rangle^{S},\left|\psi^{ \pm}\right\rangle^{p}\left|\psi^{ \pm}\right\rangle^{F}\left|\phi^{ \pm}\right\rangle^{S}$, and $\left|\psi^{ \pm}\right\rangle^{p}\left|\psi^{ \pm}\right\rangle^{F}\left|\psi^{ \pm}\right\rangle^{S}$.

\section{DISCUSSION AND SUMMARY}

With the P-S-QND and the SWAP gates, we construct our efficient hyper-EPP for the mixed hyperentangled Bell states in the polarization DOF and two longitudinalmomentum DOFs with bit-flip errors. For constructing the P-S-QND and the SWAP gates, we utilize the reflection coefficient produced by the nonlinear interaction between the single photon and the NV-cavity system. Under the resonant condition $\omega_{0}=\omega_{p}=\omega_{c}$, the reflection coefficient of the input photon is affected by the coupling strength $g$, the NV decay rate $\gamma$, and the cavity damping rate $\kappa$. If we neglect $\kappa$ and $\gamma$, the fidelities and the efficiencies of the P-S-QND and the SWAP gates can reach $100 \%$. However, in the realistic condition, the fidelities and the efficiencies are suppressed by $\gamma$ and $\kappa$. The fidelity is defined as $F=\left|\left\langle\varphi_{r} \mid \varphi_{i}\right\rangle\right|^{2}$. Here $\left|\varphi_{i}\right\rangle$ represents the final state in the ideal condition and $\left|\varphi_{r}\right\rangle$ represents the final state in the realistic condition. The efficiency is defined as $\eta=n_{\text {out }} / n_{\text {in }}$, where $n_{\text {out }}$ represents the number of the output photons and $n_{i n}$ represents the number of the input photons. For the 

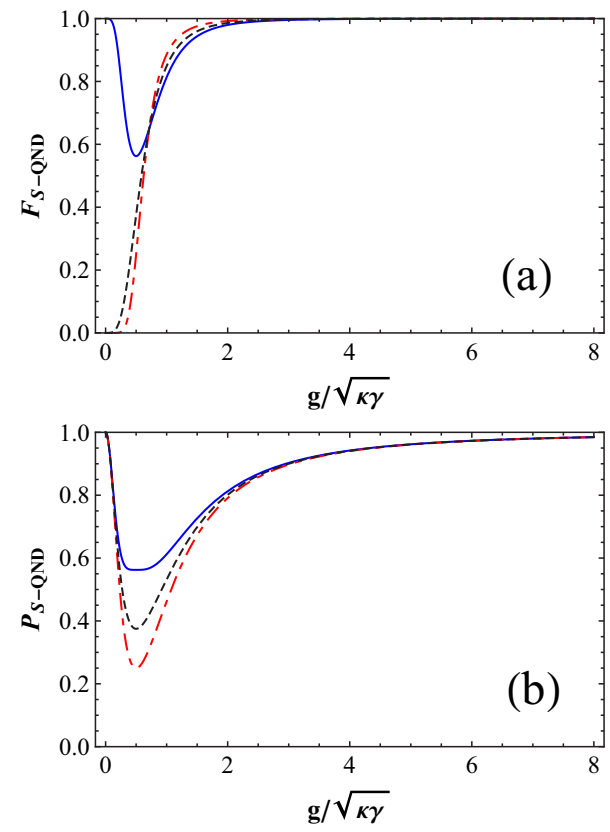

FIG. 11: (a) The fidelity of the spatial-mode-parity check QND. (b) The efficiency of the spatial-mode-parity check QND. The solid line represents $F_{1}\left(\eta_{1}\right)$ for $\left|\phi^{ \pm}\right\rangle^{p}\left|\phi^{ \pm}\right\rangle^{F}\left|\phi^{ \pm}\right\rangle^{F}$. The dash-dotted line represents $F_{S 2}\left(\eta_{S 2}\right)=F_{S 4}\left(\eta_{S 4}\right)=$ $F_{S 6}\left(\eta_{S 6}\right)=F_{S 7}\left(\eta_{S 7}\right)=F_{S 8}\left(\eta_{S 8}\right)$ for $\left|\psi^{ \pm}\right\rangle^{p}\left|\phi^{ \pm}\right\rangle^{F}\left|\phi^{ \pm}\right\rangle^{S}$, $\left|\psi^{ \pm}\right\rangle^{p}\left|\phi^{ \pm}\right\rangle^{F}\left|\psi^{ \pm}\right\rangle^{S},\left|\psi^{ \pm}\right\rangle^{p}\left|\psi^{ \pm}\right\rangle^{F}\left|\phi^{ \pm}\right\rangle^{S},\left|\phi^{ \pm}\right\rangle^{p}\left|\psi^{ \pm}\right\rangle^{F}\left|\psi^{ \pm}\right\rangle^{S}$, and $\left|\psi^{ \pm}\right\rangle^{p}\left|\psi^{ \pm}\right\rangle^{F}\left|\psi^{ \pm}\right\rangle^{S}$. The dashed line represents $F_{3}\left(\eta_{3}\right)=$ $F_{5}\left(\eta_{5}\right)$ for $\left|\phi^{ \pm}\right\rangle^{p}\left|\phi^{ \pm}\right\rangle^{F}\left|\psi^{ \pm}\right\rangle^{S}$ and $\left|\phi^{ \pm}\right\rangle^{p}\left|\psi^{ \pm}\right\rangle^{F}\left|\phi^{ \pm}\right\rangle^{S}$.

polarization parity-check QND, there exist two fidelities $F_{P i}(i=1,2)$ and two efficiencies $\eta_{p i}(i=1,2)$ corresponding to the two different polarization states $\left|\phi^{ \pm}\right\rangle^{p}$ and $\left|\psi^{ \pm}\right\rangle^{p}$. For the spatial-mode parity-check QND, there exist eight fidelities $F_{S i}(i=1 \sim 8)$ and eight efficiencies $\eta_{S i}(i=1 \sim 8)$ corresponding to the states $\left|\phi^{ \pm}\right\rangle^{p}\left|\phi^{ \pm}\right\rangle^{F}\left|\phi^{ \pm}\right\rangle^{S},\left|\psi^{ \pm}\right\rangle^{p}\left|\phi^{ \pm}\right\rangle^{F}\left|\phi^{ \pm}\right\rangle^{S},\left|\phi^{ \pm}\right\rangle^{p}\left|\phi^{ \pm}\right\rangle^{F}\left|\psi^{ \pm}\right\rangle^{S}$, $\left|\psi^{ \pm}\right\rangle^{p}\left|\phi^{ \pm}\right\rangle^{F}\left|\psi^{ \pm}\right\rangle^{S},\left|\phi^{ \pm}\right\rangle^{p}\left|\psi^{ \pm}\right\rangle^{F}\left|\phi^{ \pm}\right\rangle^{S},\left|\psi^{ \pm}\right\rangle^{p}\left|\psi^{ \pm}\right\rangle^{F}\left|\phi^{ \pm}\right\rangle^{S}$, $\left|\phi^{ \pm}\right\rangle^{p}\left|\psi^{ \pm}\right\rangle^{F}\left|\psi^{ \pm}\right\rangle^{S}$, and $\left|\psi^{ \pm}\right\rangle^{p}\left|\psi^{ \pm}\right\rangle^{F}\left|\psi^{ \pm}\right\rangle^{S}$, respectively. As $r_{0}$ is not affected by $g, \kappa$, nor $\gamma$, we have $F_{S 2}\left(\eta_{S 2}\right)=$ $F_{S 4}\left(\eta_{S 4}\right)=F_{S 6}\left(\eta_{S 6}\right)=F_{S 7}\left(\eta_{S 7}\right)=F_{S 8}\left(\eta_{S 8}\right)$ and $F_{S 3}\left(\eta_{S 3}\right)=F_{S 5}\left(\eta_{S 5}\right)$. The fidelities and the efficiencies (discussed the detail in the Appendix) of the polarization parity-check QND, spatial parity-check QND, and the PP-SWAP gate, which vary with the parameter $g / \sqrt{\kappa \gamma}$, are shown in Fig. 10, Figs. 11, and 12, respectively.

By far, several groups have experimentally demonstrated the coupling between a diamond NV center and a microcavity [49 52]. In 2009, Barclay et al. [49] reported their experiment with the parameters $\left[g, \kappa, \gamma_{t o t}, \gamma\right] / 2 \pi=$ $[0.30,26,0.013,0.0004] G H z$ for coupling the diamond NV centers to a chip-based microcavity. This experiment is implemented in a weak coupling regime with a low- $Q$ factor. In their experiment, the NV center coherent coupling rate within the narrow-band zero phonon line (ZPL) is almost one-third of the total coupling rate. We have $r=$ 0.94 at $\omega_{0}=\omega_{p}=\omega_{c}$. Based on their experimental pa-
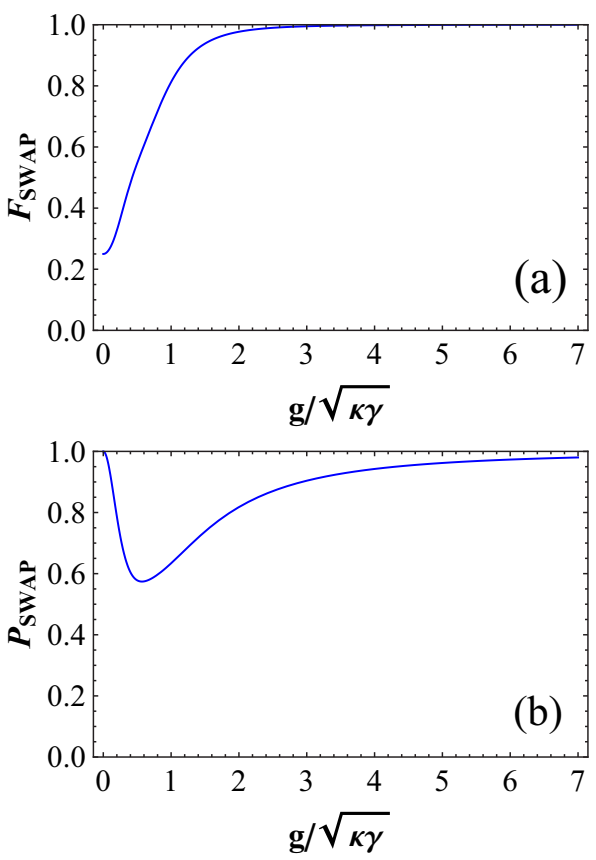

FIG. 12: (a) The fidelity of the P-P-SWAP gate. (b) The efficiency of the P-P-SWAP gate.

rameters, the fidelities and the efficiencies of our polarization parity-check QND can reach $F_{p 1,2}=99.76 \%, 99.91 \%$ and $\eta_{p 1,2}=94.84 \%, 94.54 \%$, respectively. The fidelities and the efficiencies of our spatial-mode parity-check QND can reach $F_{S 1,2,3}=99.53 \%, 99.83 \%, 99.68 \%$ and $\eta_{S 1,2,3}=89.95 \%, 89.38 \%, 89.66 \%$, respectively. The fidelity and the efficiency of the P-P-SWAP gate can reach $F_{S W A P}=99.46 \%$ and $\eta_{S W A P}=90.08 \%$, respectively.

In our proposal, the two cavity modes with right- and left- circular polarizations, which couple to the two transitions $|+\rangle \leftrightarrow\left|A_{2}\right\rangle$ and $|-\rangle \leftrightarrow\left|A_{2}\right\rangle$ respectively, are required. Many good experiments that provide a cavity supporting both of two circularly-polarized modes with the same frequency have been realized [57 63]. For example, Luxmoore et al. [57] presented a technique for fine tuning of the energy split between the two circularlypolarized modes to just $0.15 \mathrm{~nm}$ in 2012 .

In summary, we have proposed a hyper-EPP for the mixed hyperentangled Bell states with bit-flip errors in three DOFs, including the polarization and two longitudinal-momentum DOFs. Our hyper-EPP is completed with one or two steps. In the first step, Alice and Bob perform the P-S-QNDs and Hadamard operations on their own photons. If the states of the two photon pairs are projected into the states in case (1), the hyperentanglement purification process is accomplished and it costs one photon pair to purify another one. If the states of the two photon pairs are projected into cases (3), (4), (5), (6), (7) and (8), the second step of our hyper-EPP is needed. In the second step, Alice and Bob swap the states between two-photon systems in the same DOF or between different DOFs of the one photon by the method 
of combining the P-P-SWAP gate, the P-F-SWAP gate, and the P-S-SWAP gate. If the hyperentanglement purification process is accomplished with two steps, it costs another three pairs to purify one photon pair. We have showed the feasibility of our hyper-EPP as high fidelities and efficiencies of P-S-QND and SWAP gates can be obtained based on the existing experimental parameters and there exists the cavity that supports two circularlypolarized modes with the same frequency.

\section{ACKNOWLEDGEMENTS}

This work was supported by the National Natural Science Foundation of China under Grant No. 11674033 and No. 11474026, and the Fundamental Research Funds for the Central Universities under Grant No. 2015KJJCA01.

\section{APPENDIX}

A. Case (2) in the first step of our hyper-EPP with SWAP gates

In case (2), the two identical two-photon systems $A C$ and $B D$ are in different parity-mode in all of the three DOFs, including the polarization DOF, the first-longitudinal-momentum DOF, and the secondlongitudinal- momentum DOF.

When the photons $A C$ are in the even-parity modes and $B D$ are in the odd-parity modes at the same time in the polarization DOF, in the first-longitudinalmomentum DOF, and in the second-longitudinalmomentum DOF, Alice and Bob obtain the states

$$
\begin{aligned}
\left|\Phi_{5}\right\rangle^{p} & =\frac{1}{\sqrt{2}}(|R R R L\rangle+|L L L R\rangle)_{A B C D}, \\
\left|\Phi_{6}\right\rangle^{p} & =\frac{1}{\sqrt{2}}(|R L R R\rangle+|L R L L\rangle)_{A B C D}, \\
\left|\Phi_{5}\right\rangle^{F} & =\frac{1}{\sqrt{2}}(|r r r l\rangle+|l l l r\rangle)_{A B C D}, \\
\left|\Phi_{6}\right\rangle^{F} & =\frac{1}{\sqrt{2}}(|r l r r\rangle+|l r l l\rangle)_{A B C D}, \\
\left|\Phi_{5}\right\rangle^{S} & =\frac{1}{\sqrt{2}}(|E E E I\rangle+|I I I E\rangle)_{A B C D}, \\
\left|\Phi_{6}\right\rangle^{S} & =\frac{1}{\sqrt{2}}(|E I E E\rangle+|I E I I\rangle)_{A B C D} .
\end{aligned}
$$

On the contrary, when the photons $A C$ are in the odd-parity modes and $B D$ are in the even-parity modes at the same time in the polarization DOF, in the first-longitudinal-momentum DOF or in the secondlongitudinal-momentum DOF, Alice and Bob obtain the states

$$
\begin{aligned}
\left|\Phi_{7}\right\rangle^{p} & =\frac{1}{\sqrt{2}}(|R R L R\rangle+|L L R L\rangle)_{A B C D}, \\
\left|\Phi_{8}\right\rangle^{p} & =\frac{1}{\sqrt{2}}(|R L L L\rangle+|L R R R\rangle)_{A B C D}, \\
\left|\Phi_{7}\right\rangle^{F} & =\frac{1}{\sqrt{2}}(|r r l r\rangle+|l l r l\rangle)_{A B C D}, \\
\left|\Phi_{8}\right\rangle^{F} & =\frac{1}{\sqrt{2}}(|r l l l\rangle+|l r r r\rangle)_{A B C D}, \\
\left|\Phi_{7}\right\rangle^{S} & =\frac{1}{\sqrt{2}}(|E E I E\rangle+|I I E I\rangle)_{A B C D}, \\
\left|\Phi_{8}\right\rangle^{S} & =\frac{1}{\sqrt{2}}(|E I I I\rangle+|I E E E\rangle)_{A B C D} .
\end{aligned}
$$

The states $\left|\Phi_{7}\right\rangle^{p},\left|\Phi_{8}\right\rangle^{p},\left|\Phi_{7}\right\rangle^{F},\left|\Phi_{8}\right\rangle^{F},\left|\Phi_{7}\right\rangle^{S}$, and $\left|\Phi_{8}\right\rangle^{S}$ can be transformed into states $\left|\Phi_{5}\right\rangle^{p},\left|\Phi_{6}\right\rangle^{p},\left|\Phi_{5}\right\rangle^{F},\left|\Phi_{6}\right\rangle^{F}$, $\left|\Phi_{5}\right\rangle^{S}$, and $\left|\Phi_{6}\right\rangle^{S}$, respectively.

Next, Alice and Bob perform Hadamard operations in the polarization and the two longitudinal-momentum DOFs on photons $C$ and $D$, respectively. In the polarization DOF, the states $\left|\Phi_{5}\right\rangle^{p}$ and $\left|\Phi_{6}\right\rangle^{p}$ are transformed into the states $\left|\Phi_{5^{\prime}}\right\rangle^{p}$ and $\left|\Phi_{6^{\prime}}\right\rangle^{p}$, respectively. In the first-longitudinal-momentum DOF, the states $\left|\Phi_{5}\right\rangle^{F}$ and $\left|\Phi_{6}\right\rangle^{F}$ are transformed into $\left|\Phi_{5}^{\prime}\right\rangle^{F}$ and $\left|\Phi_{6}^{\prime}\right\rangle^{F}$, respectively. In the second-longitudinal-momentum DOF, the states $\left|\Phi_{5}\right\rangle^{S}$ and $\left|\Phi_{6}\right\rangle^{S}$ are transformed into $\left|\Phi_{5}^{\prime}\right\rangle^{S}$ and $\left|\Phi_{6}^{\prime}\right\rangle^{S}$, respectively. Here

$$
\begin{aligned}
\left|\Phi_{5}^{\prime}\right\rangle^{p}= & \frac{1}{2 \sqrt{2}}\left[(|R R\rangle+|L L\rangle)_{A B}(|R R\rangle-|L L\rangle)_{C D}\right. \\
& \left.+(|R R\rangle-|L L\rangle)_{A B}(-|R L\rangle+|L R\rangle)_{C D}\right], \\
\left|\Phi_{6}^{\prime}\right\rangle^{p}= & \frac{1}{2 \sqrt{2}}\left[(|R L\rangle+|L R\rangle)_{A B}(|R R\rangle+|L L\rangle)_{C D}\right. \\
& \left.+(-|R L\rangle+|L R\rangle)_{A B}(-|R L\rangle-|L R\rangle)_{C D}\right], \\
\left|\Phi_{5}^{\prime}\right\rangle^{F}= & \frac{1}{2 \sqrt{2}}\left[(|r r\rangle+|l l\rangle)_{A B}(|r r\rangle-|l l\rangle)_{C D}\right. \\
& \left.+(|r r\rangle-|l l\rangle)_{A B}(-|r l\rangle+|l r\rangle)_{C D}\right], \\
\left|\Phi_{6}^{\prime}\right\rangle^{F}= & \frac{1}{2 \sqrt{2}}\left[(|r l\rangle+|l r\rangle)_{A B}(|r r\rangle+|l l\rangle)_{C D}\right. \\
& \left.+(-|r l\rangle+|l r\rangle)_{A B}(-|r l\rangle-|l r\rangle)_{C D}\right], \\
\left|\Phi_{5}^{\prime}\right\rangle^{S}= & \frac{1}{2 \sqrt{2}}\left[(|E E\rangle+|I I\rangle)_{A B}(|E E\rangle-|I I\rangle)_{C D}\right. \\
& \left.+(|E E\rangle-|I I\rangle)_{A B}(-|E I\rangle+|I E\rangle)_{C D}\right], \\
\left|\Phi_{6}^{\prime}\right\rangle^{S}= & \frac{1}{2 \sqrt{2}}\left[(|E I\rangle+|I E\rangle)_{A B}(|E E\rangle+|I I\rangle)_{C D}\right. \\
& \left.+(-|E I\rangle+|I E\rangle)_{A B}(-|E I\rangle-|I E\rangle)_{C D}\right] .
\end{aligned}
$$

Finally, the photons $C$ and $D$ are detected by singlephoton detectors, respectively. If the photons $C D$ are in the even-parity mode in the polarization DOF (the first-longitudinal-momentum DOF or the secondlongitudinal-momentum DOF), nothing is needed to be 
done. If the photons $C D$ are in the odd-parity mode in the polarization DOF (the first-longitudinal-momentum DOF or the second-longitudinal-momentum DOF), the phase-flip operation $\sigma_{z}^{p}=|R\rangle\langle R|-| L\rangle\langle L|\left(\sigma_{z}^{F}=|r\rangle\langle r|-\right.$ $|l\rangle\langle l|$ or $\left.\sigma_{z}^{S}=|E\rangle\langle E|-| I\rangle\langle I|\right)$ is operated on the photon $B$. If $A C$ and $B D$ are projected into this case, Alice and Bob will discard the two photon pairs.

\section{B. The complete second step of our hyper-EPP with SWAP gates}

(a) If the two two-photon systems $A B$ and $C D$ are projected into the states in case (3) in the first step, Alice and Bob can seek another two two-photon systems $A^{\prime} B^{\prime}$ and $C^{\prime} D^{\prime}$ which are projected into the states in case (8). The P-P-SWAP gate shown in Fig. 5 can be used to complete the second step of our hyper-EPP. The states of the two photon pairs $A B$ and $A^{\prime} B^{\prime}$ are transformed into the states

$$
\begin{aligned}
& |\Phi\rangle_{A B 5}=\left|\phi^{+}\right\rangle^{p}\left|\phi^{+}\right\rangle^{F}\left|\phi^{+}\right\rangle^{S}, \\
& |\Phi\rangle_{A^{\prime} B^{\prime} 5}=\left|\psi^{+}\right\rangle^{p}\left|\psi^{+}\right\rangle^{F}\left|\psi^{+}\right\rangle^{S} .
\end{aligned}
$$

Here $|\Phi\rangle_{A B 5}$ and $|\Phi\rangle_{A^{\prime} B^{\prime} 5}$ are the finale states of case (a) in the second step of our hyper-EPP.

(b) If the two two-photon systems $A B$ and $C D$ are projected into the states in case (4) in the first step, Alice and Bob can seek another two two-photon systems $A^{\prime} B^{\prime}$ and $C^{\prime} D^{\prime}$ which are projected into the states in case $(7)$. The P-P-SWAP gate shown in Fig. 5] and the P-F-SWAP gate shown in Fig. 6(a) can be used to complete the second step of our hyper-EPP.

First, Alice swaps the polarization states and the firstlongitudinal-momentum states of photons $A$ and performs the same operations on photon $A^{\prime}$. Bob performs the same operations on photons $B$ and $B^{\prime}$, respectively. Second, Alice swaps the polarization states between the photons $A$ and $A^{\prime}$. Bob swaps the polarization states between the photons $B$ and $B^{\prime}$. Finally, Alice and Bob swap the polarization states and the first-longitudinalmomentum states on the photons $A, B, A^{\prime}$ and $B^{\prime}$ again. After these operations, the states of photon pairs become

$$
\begin{aligned}
& |\Phi\rangle_{A B 6}=\left|\psi^{+}\right\rangle^{p}\left|\psi^{+}\right\rangle^{F}\left|\phi^{+}\right\rangle^{S}, \\
& |\Phi\rangle_{A^{\prime} B^{\prime} 6}=\left|\phi^{+}\right\rangle^{p}\left|\phi^{+}\right\rangle^{F}\left|\psi^{+}\right\rangle^{S} .
\end{aligned}
$$

Here $|\Phi\rangle_{A B 6}$ and $|\Phi\rangle_{A^{\prime} B^{\prime} 6}$ are the final states of case (b) in the second step of our hyper-EPP.

(c) If the two two-photon systems $A B$ and $C D$ are projected into the states in case (5) in the first step, Alice and Bob can seek another two two-photon systems $A^{\prime} B^{\prime}$ and $C^{\prime} D^{\prime}$ which are projected into the states in case (6). Alice and Bob perform the similar operations as what they do in case (b) in the second step. The difference is that in this case, they use the P-S-SWAP gate shown in Fig. 6 (b), instead of the P-F-SWAP gate. The final states of case (c) in the second step of our hyper-EPP are

$$
\begin{aligned}
& |\Phi\rangle_{A B 7}=\left|\psi^{+}\right\rangle^{p}\left|\phi^{+}\right\rangle^{F}\left|\psi^{+}\right\rangle^{S}, \\
& |\Phi\rangle_{A^{\prime} B^{\prime} 7}=\left|\phi^{+}\right\rangle^{p}\left|\psi^{+}\right\rangle^{F}\left|\phi^{+}\right\rangle^{S} .
\end{aligned}
$$

(d) If the two two-photon systems $A B$ and $C D$ are projected into the states in case (7) in the first step, Alice and Bob can seek another two two-photon systems $A^{\prime} B^{\prime}$ and $C^{\prime} D^{\prime}$ which are projected into the states in case (4). The second step of our hyper-EPP is completed with the P-P-SWAP gates and the P-S-SWAP gates.

First, Alice and Bob swap the polarization states of the two two-photon system $A B$ and the states of the system $A^{\prime} B^{\prime}$. Second, Alice and Bob swap the polarization states and the second longitudinal-momentum states of photons $A, B, A^{\prime}$, and $B^{\prime}$, respectively. Third, Alice and Bob swap the polarization states of the two systems $A B$ and $A^{\prime} B^{\prime}$ again. Finally, Alice and Bob swap the polarization states of the second-longitudinal-momentum states of photons $A, B, A^{\prime}$, and $B^{\prime}$, respectively. The states of the two systems are changed to be

$$
\begin{aligned}
& |\Phi\rangle_{A B 8}=\left|\phi^{+}\right\rangle^{p}\left|\phi^{+}\right\rangle^{F}\left|\psi^{+}\right\rangle^{S} \\
& |\Phi\rangle_{A^{\prime} B^{\prime} 8}=\left|\psi^{+}\right\rangle^{p}\left|\psi^{+}\right\rangle^{F}\left|\phi^{+}\right\rangle^{S} .
\end{aligned}
$$

Here $|\Phi\rangle_{A B 8}$ and $|\Phi\rangle_{A^{\prime} B^{\prime} 8}$ are the final states of this case in the second step of our hyper-EPP.

(e) If the two two-photon systems $A B$ and $C D$ are projected into the states in case (8) in the first step, Alice and Bob can seek another two two-photon systems $A^{\prime} B^{\prime}$ and $C^{\prime} D^{\prime}$ which are projected into the states in case (3). The second step of our hyper-EPP is completed with the P-P-SWAP gates, P-F-SWAP gates, and P-S-SWAP gates.

First, Alice and Bob swap the polarization states and the first-longitudinal-momentum states of photons $A, B$, $A^{\prime}$, and $B^{\prime}$ by the P-F-SWAP gate, respectively. Second, Alice and Bob swap the polarization states between system $A B$ and system $A^{\prime} B^{\prime}$ with the P-P-SWAP gate. Third, Alice and Bob swap the polarization states and the first-longitudinal-momentum states of photons $A, B$, $A^{\prime}$, and $B^{\prime}$ again with the P-F-SWAP gate, respectively. Fourth, Alice and Bob swap the polarization states and the second-longitudinal-momentum states of photons $A$, $B, A^{\prime}$, and $B^{\prime}$ with the P-S-SWAP gate, respectively. Fifth, Alice and Bob swap the polarization states between system $A B$ and system $A^{\prime} B^{\prime}$ by the P-P-SWAP gate for the third time. Finally, Alice and Bob swap the polarization states and the second-longitudinal-momentum states of photons $A, B, A^{\prime}$, and $B^{\prime}$ respectively again. Thus, the states of systems become

$$
\begin{aligned}
& |\Phi\rangle_{A B 9}=\left|\psi^{+}\right\rangle^{p}\left|\psi^{+}\right\rangle^{F}\left|\psi^{+}\right\rangle^{S} \\
& |\Phi\rangle_{A^{\prime} B^{\prime} 9}=\left|\phi^{+}\right\rangle^{p}\left|\phi^{+}\right\rangle^{F}\left|\phi^{+}\right\rangle^{S} .
\end{aligned}
$$

Here $|\Phi\rangle_{A B 9}$ and $|\Phi\rangle_{A^{\prime} B^{\prime} 9}$ are the final states of this case in the second step of our hyper-EPP. 


\section{The fidelity and the efficiency}

There exist two fidelities $F_{P 1,2}$ and efficiencies $\eta_{P 1,2}$ of the polarization parity-check QND. The fidelity (efficiency) $F_{P 1}\left(\eta_{P 1}\right)$ corresponds to the states $\left|\phi^{ \pm}\right\rangle^{p}\left|\phi^{ \pm}\right\rangle^{F}\left|\phi^{ \pm}\right\rangle^{S},\left|\phi^{ \pm}\right\rangle^{p}\left|\phi^{ \pm}\right\rangle^{F}\left|\psi^{ \pm}\right\rangle^{S},\left|\phi^{ \pm}\right\rangle^{p}\left|\psi^{ \pm}\right\rangle^{F}\left|\phi^{ \pm}\right\rangle^{S}$, and $\left|\phi^{ \pm}\right\rangle^{p}\left|\psi^{ \pm}\right\rangle^{F}\left|\psi^{ \pm}\right\rangle^{S}$. The fidelity (efficiency) $F_{P 2}\left(\eta_{P 2}\right)$ corresponds to the states $\left|\psi^{ \pm}\right\rangle^{p}\left|\phi^{ \pm}\right\rangle^{F}\left|\phi^{ \pm}\right\rangle^{S},\left|\psi^{ \pm}\right\rangle^{p}\left|\phi^{ \pm}\right\rangle^{F}\left|\psi^{ \pm}\right\rangle^{S},\left|\psi^{ \pm}\right\rangle^{p}\left|\psi^{ \pm}\right\rangle^{F}\left|\phi^{ \pm}\right\rangle^{S}$, and $\left|\psi^{ \pm}\right\rangle^{p}\left|\psi^{ \pm}\right\rangle^{F}\left|\psi^{ \pm}\right\rangle^{S}$. The fidelities of the polarization parity-check QND can be expressed as

$$
F_{P 1}=\frac{\left|2+r^{2}+r_{0}^{2}\right|^{2}}{4\left[2+\left|r^{2}\right|^{2}+\left|r_{0}^{2}\right|^{2}\right]}, \quad F_{P 2}=\frac{\left|r-r_{0}\right|^{2}}{2\left[|r|^{2}+\left|r_{0}\right|^{2}\right]} .
$$

The efficiencies of the polarization parity-check QND can be expressed as

$$
\eta_{p 1}=\frac{1}{4}\left[2+\left|r^{2}\right|^{2}+\left|r_{0}^{2}\right|^{2}\right], \quad \eta_{P 2}=\frac{1}{2}\left[\left|r_{0}\right|^{2}+|r|^{2}\right] .
$$

The fidelity and the efficiency of the polarization parity-check QND, which vary with the parameter $g / \sqrt{\kappa \gamma}$, are shown in Figs. 9(a) and 9(b), respectively.

For the spatial-mode parity-check QND, there exist eight fidelities $F_{S i}(i=1,2, \cdots, 8)$ and eight efficiencies $\eta_{S i}(i=$ $1,2, \cdots, 8)$ corresponding to the states $\left|\phi^{ \pm}\right\rangle^{p}\left|\phi^{ \pm}\right\rangle^{F}\left|\phi^{ \pm}\right\rangle^{F},\left|\psi^{ \pm}\right\rangle^{p}\left|\phi^{ \pm}\right\rangle^{F}\left|\phi^{ \pm}\right\rangle^{F},\left|\phi^{ \pm}\right\rangle^{p}\left|\phi^{ \pm}\right\rangle^{F}\left|\psi^{ \pm}\right\rangle^{F},\left|\psi^{ \pm}\right\rangle^{p}\left|\phi^{ \pm}\right\rangle^{F}\left|\psi^{ \pm}\right\rangle^{F}$, $\left|\phi^{ \pm}\right\rangle^{p}\left|\psi^{ \pm}\right\rangle^{F}\left|\phi^{ \pm}\right\rangle^{F},\left|\psi^{ \pm}\right\rangle^{p}\left|\psi^{ \pm}\right\rangle^{F}\left|\phi^{ \pm}\right\rangle^{F},\left|\phi^{ \pm}\right\rangle^{p}\left|\psi^{ \pm}\right\rangle^{F}\left|\psi^{ \pm}\right\rangle^{F}$, and $\left|\psi^{ \pm}\right\rangle^{p}\left|\psi^{ \pm}\right\rangle^{F}\left|\psi^{ \pm}\right\rangle^{F}$, respectively. The fidelities of the spatial-mode parity-check QND can be expressed as

$$
\begin{aligned}
F_{S 1} & =\frac{\left|\left(r^{2}+r_{0}^{2}\right)^{2}+4\left(r^{2}+r_{0}^{2}\right)+4\right|^{2}}{16\left[4\left(\left|r^{2}\right|^{2}+\left|r_{0}^{2}\right|^{2}\right)+\left(\left|r^{4}\right|^{2}+2\left|r^{2} r_{0}^{2}\right|^{2}+\left|r_{0}^{4}\right|^{2}\right)+4\right]}, \\
F_{S 2} & =\frac{\left|r^{2} r_{0}^{2}-2 r r_{0}+1\right|^{2}}{4\left[\left|r^{2} r_{0}^{2}\right|^{2}+2\left|r r_{0}\right|^{2}+1\right]}, \\
F_{S 3} & =\frac{\left|\left(r^{2}+r_{0}^{2}\right)\left(r-r_{0}\right)+2\left(r-r_{0}\right)\right|^{2}}{8\left[\left(\left|r^{3}\right|^{2}+\left|r_{0}^{3}\right|^{2}+\left|r_{0} r^{2}\right|^{2}+\left|r_{0}^{2} r\right|^{2}\right)+2\left(|r|^{2}+\left|r_{0}\right|^{2}\right)\right]}, \\
F_{S 4} & =\frac{\left|\left(r-r_{0}\right)\left(1-r r_{0}\right)\right|^{2}}{4\left[\left|r r_{0}^{2}\right|^{2}+\left|r_{0} r^{2}\right|^{2}+|r|^{2}+\left|r_{0}\right|^{2}\right]}, \\
F_{S 5} & =\frac{\left|\left(r^{2}+r_{0}^{2}\right)\left(r-r_{0}\right)+2\left(r-r_{0}\right)\right|^{2}}{8\left[\left(\left|r^{3}\right|^{2}+\left|r r_{0}^{2}\right|^{2}+\left|r_{0} r^{2}\right|^{2}+\left|r_{0}^{3}\right|^{2}\right)+2\left(|r|^{2}+\left|r_{0}\right|^{2}\right)\right]}, \\
F_{S 6} & =\frac{\left|\left(r-r_{0}\right)\left(1-r r_{0}\right)\right|^{2}}{4\left[|r|^{2}+\left|r_{0}\right|^{2}+\left|r r_{0}^{2}\right|^{2}+\left|r_{0} r^{2}\right|^{2}\right]}, \\
F_{S 7} & =\frac{\left|r^{2}+r_{0}^{2}-2 r r_{0}\right|^{2}}{4\left[\left|r^{2}\right|^{2}+2\left|r r_{0}\right|^{2}+\left|r_{0}^{2}\right|^{2}\right]}, \\
F_{S 8} & =\frac{\left|r^{2}+r_{0}^{2}-2 r r_{0}\right|^{2}}{4\left[\left|r^{2}\right|^{2}+2\left|r r_{0}\right|^{2}+\left|r_{0}^{2}\right|^{2}\right]} .
\end{aligned}
$$

The efficiencies of the spatial-mode parity-check QND can be expressed as

$$
\begin{aligned}
& \eta_{S 1}=\frac{1}{16}\left[4\left(\left|r^{2}\right|^{2}+\left|r_{0}^{2}\right|^{2}\right)+\left(\left|r^{4}\right|^{2}+2\left|r^{2} r_{0}^{2}\right|^{2}+\left|r_{0}^{4}\right|^{2}\right)+4\right], \\
& \eta_{S 2}=\frac{1}{4}\left[\left|r^{2} r_{0}^{2}\right|^{2}+2\left|r r_{0}\right|^{2}+1\right], \\
& \eta_{S 3}=\frac{1}{8}\left[\left(\left|r^{3}\right|^{2}+\left|r_{0}^{3}\right|^{2}+\left|r_{0} r^{2}\right|^{2}+\left|r_{0}^{2} r\right|^{2}\right)+2\left(|r|^{2}+\left|r_{0}\right|^{2}\right)\right], \\
& \eta_{S 4}=\frac{1}{4}\left[\left|r r_{0}^{2}\right|^{2}+\left|r_{0} r^{2}\right|^{2}+|r|^{2}+\left|r_{0}\right|^{2}\right], \\
& \eta_{S 5}=\frac{1}{8}\left[\left(\left|r^{3}\right|^{2}+\left|r r_{0}^{2}\right|^{2}+\left|r_{0} r^{2}\right|^{2}+\left|r_{0}^{3}\right|^{2}\right)+2\left(|r|^{2}+\left|r_{0}\right|^{2}\right)\right], \\
& \eta_{S 6}=\frac{1}{4}\left[|r|^{2}+\left|r_{0}\right|^{2}+\left|r r_{0}^{2}\right|^{2}+\left|r_{0} r^{2}\right|^{2}\right], \\
& \eta_{S 7}=\frac{1}{4}\left[\left|r^{2}\right|^{2}+2\left|r r_{0}\right|^{2}+\left|r_{0}^{2}\right|^{2}\right], \\
& \eta_{S 8}=\frac{1}{4}\left[\left|r^{2}\right|^{2}+2\left|r r_{0}\right|^{2}+\left|r_{0}^{2}\right|^{2}\right] .
\end{aligned}
$$


As $r_{0}=-1$ is an exact value, we have $F_{S 2}\left(\eta_{S 2}\right)=F_{S 4}\left(\eta_{S 4}\right)=F_{S 6}\left(\eta_{S 6}\right)=F_{S 7}\left(\eta_{S 7}\right)=F_{S 8}\left(\eta_{S 8}\right)$ and $F_{S 3}\left(\eta_{S 3}\right)=$ $F_{S 5}\left(\eta_{S 5}\right)$. The fidelities and the efficiencies of the spatial-mode parity-check QND, which vary with the parameter $g / \sqrt{\kappa \gamma}$, are shown in Figs. 10(a) and 10(b), respectively.

The fidelity and the efficiency of the P-P-SWAP gate proposed in Sec.IV(A) can be expressed as

$$
\begin{aligned}
F_{S W A P}^{\prime}= & \frac{\left|f_{1}^{\prime}+f_{2}^{\prime}+f_{3}^{\prime}+f_{4}^{\prime}+f_{4}^{\prime}+f_{5}^{\prime}+f_{6}^{\prime}+f_{7}^{\prime}+f_{8}^{\prime}\right|^{2}}{\eta_{S W A P}^{\prime}}, \\
\eta_{S W A P}^{\prime}= & \frac{1}{32}\left[\left|2 \alpha_{1} \alpha_{2} r^{2}+\left(\alpha_{1} \beta_{2}+\beta_{1} \alpha_{2}\right)\left(r^{2} r_{0}+r^{3}+r_{0}^{3}-r_{0}^{2} r\right)+\beta_{1} \beta_{2}\left(r^{4}+r_{0}^{4}\right)\right|^{2}\right. \\
& +\left|2 \alpha_{1} \alpha_{2} r^{2}+\left(\alpha_{1} \beta_{2}+\beta_{1} \alpha_{2}\right)\left(r^{2} r_{0}+r^{3}-r_{0}^{3}+r_{0}^{2} r\right)+\beta_{1} \beta_{2}\left(r^{4}-r_{0}^{4}+2 r^{2} r_{0}^{2}\right)\right|^{2} \\
& +\left|2 \alpha_{1} \alpha_{2} r-\left(\alpha_{1} \beta_{2}-\beta_{1} \alpha_{2}\right)\left(r^{2}+r_{0}^{2}\right)-\beta_{1} \beta_{2}\left(r r_{0}^{2}+r^{3}+r_{0}^{3}-r_{0} r^{2}\right)\right|^{2} \\
& +\left|2 \alpha_{1} \alpha_{2} r-\left(\alpha_{1} \beta_{2}-\beta_{1} \alpha_{2}\right)\left(2 r r_{0}+r^{2}-r_{0}^{2}\right)-\beta_{1} \beta_{2}\left(r r_{0}^{2}+r^{3}-r_{0}^{3}+r_{0} r^{2}\right)\right|^{2} \\
& +\left|2 \alpha_{1} \alpha_{2} r+\left(\alpha_{1} \beta_{2}-\beta_{1} \alpha_{2}\right)\left(r^{2}+r_{0}^{2}\right)-\beta_{1} \beta_{2}\left(r r_{0}^{2}+r^{3}+r_{0}^{3}-r_{0} r^{2}\right)\right|^{2} \\
& +\left|2 \alpha_{1} \alpha_{2} r+\left(\alpha_{1} \beta_{2}-\beta_{1} \alpha_{2}\right)\left(2 r r_{0}+r^{2}-r_{0}^{2}\right)-\beta_{1} \beta_{2}\left(r r_{0}^{2}+r^{3}-r_{0}^{3}+r_{0} r^{2}\right)\right|^{2} \\
& +\left|2 \alpha_{1} \alpha_{2}-2\left(\alpha_{1} \beta_{2}+\beta_{1} \alpha_{2}\right) r_{0}+2 \beta_{1} \beta_{2} r_{0}^{2}\right|^{2} \\
& \left.+\left|2 \alpha_{1} \alpha_{2}-2\left(\alpha_{1} \beta_{2}+\beta_{1} \alpha_{2}\right) r+2 \beta_{1} \beta_{2} r^{2}\right|^{2}\right] .
\end{aligned}
$$

Here,

$$
\begin{aligned}
& f_{1}^{\prime}=\frac{1}{16}\left[2 \alpha_{1} \alpha_{2} r^{2}+\left(\alpha_{1} \beta_{2}+\beta_{1} \alpha_{2}\right)\left(r^{2} r_{0}+r^{3}+r_{0}^{3}-r_{0}^{2} r\right)+\beta_{1} \beta_{2}\left(r^{4}+r_{0}^{4}\right)\right]\left(\alpha_{1}-\beta_{1}\right)\left(\alpha_{2}-\beta_{2}\right), \\
& f_{2}^{\prime}=\frac{1}{16}\left[2 \alpha_{1} \alpha_{2} r^{2}+\left(\alpha_{1} \beta_{2}+\beta_{1} \alpha_{2}\right)\left(r^{2} r_{0}+r^{3}-r_{0}^{3}+r_{0}^{2} r\right)+\beta_{1} \beta_{2}\left(r^{4}-r_{0}^{4}+2 r^{2} r_{0}^{2}\right)\right]\left(\alpha_{1}+\beta_{1}\right)\left(\alpha_{2}+\beta_{2}\right), \\
& f_{3}^{\prime}=\frac{1}{16}\left[2 \alpha_{1} \alpha_{2} r-\left(\alpha_{1} \beta_{2}-\beta_{1} \alpha_{2}\right)\left(r^{2}+r_{0}^{2}\right)-\beta_{1} \beta_{2}\left(r r_{0}^{2}+r^{3}+r_{0}^{3}-r_{0} r^{2}\right)\right]\left(\alpha_{1}+\beta_{1}\right)\left(\alpha_{2}-\beta_{2}\right), \\
& f_{4}^{\prime}=\frac{1}{16}\left[2 \alpha_{1} \alpha_{2} r-\left(\alpha_{1} \beta_{2}-\beta_{1} \alpha_{2}\right)\left(2 r r_{0}+r^{2}-r_{0}^{2}\right)-\beta_{1} \beta_{2}\left(r r_{0}^{2}+r^{3}-r_{0}^{3}+r_{0} r^{2}\right)\right]\left(\alpha_{1}-\beta_{1}\right)\left(\alpha_{2}+\beta_{2}\right), \\
& f_{5}^{\prime}=\frac{1}{16}\left[2 \alpha_{1} \alpha_{2} r+\left(\alpha_{1} \beta_{2}-\beta_{1} \alpha_{2}\right)\left(r^{2}+r_{0}^{2}\right)-\beta_{1} \beta_{2}\left(r r_{0}^{2}+r^{3}+r_{0}^{3}-r_{0} r^{2}\right)\right]\left(\alpha_{1}-\beta_{1}\right)\left(\alpha_{2}+\beta_{2}\right), \\
& f_{6}^{\prime}=\frac{1}{16}\left[2 \alpha_{1} \alpha_{2} r+\left(\alpha_{1} \beta_{2}-\beta_{1} \alpha_{2}\right)\left(2 r r_{0}+r^{2}-r_{0}^{2}\right)-\beta_{1} \beta_{2}\left(r r_{0}^{2}+r^{3}-r_{0}^{3}+r_{0} r^{2}\right)\right]\left(\alpha_{1}+\beta_{1}\right)\left(\alpha_{2}-\beta_{2}\right), \\
& f_{7}^{\prime}=\frac{1}{16}\left[2 \alpha_{1} \alpha_{2}-2\left(\alpha_{1} \beta_{2}+\beta_{1} \alpha_{2}\right) r_{0}+2 \beta_{1} \beta_{2} r_{0}^{2}\right]\left(\alpha_{1}+\beta_{1}\right)\left(\alpha_{2}+\beta_{2}\right), \\
& f_{8}^{\prime}=\frac{1}{16}\left[2 \alpha_{1} \alpha_{2}-2\left(\alpha_{1} \beta_{2}+\beta_{1} \alpha_{2}\right) r+2 \beta_{1} \beta_{2} r^{2}\right]\left(\alpha_{1}-\beta_{1}\right)\left(\alpha_{2}-\beta_{2}\right) .
\end{aligned}
$$

In our hyper-EPP, our P-P-SWAP gate is used to swap the maximally entangled states between two photon pairs, and $\alpha_{1}=\beta_{1}=\alpha_{2}=\beta_{2}$. Thus, the fidelity and efficiency of the P-P-SWAP gate can be expressed as

$$
\begin{aligned}
F_{S W A P}= & \frac{\left|f_{1}+f_{2}\right|^{2}}{\eta_{S W A P}}, \\
\eta_{S W A P}= & \frac{1}{32}\left[\left|r^{2}+r^{2} r_{0}+r^{3}+r_{0}^{3}-r_{0}^{2} r+\frac{1}{2}\left(r^{4}+r_{0}^{4}\right)\right|^{2}\right. \\
& +\left|r^{2}+r^{2} r_{0}+r^{3}-r_{0}^{3}+r_{0}^{2} r+\frac{1}{2}\left(r^{4}-r_{0}^{4}+2 r^{2} r_{0}^{2}\right)\right|^{2} \\
& +2\left|r-\frac{1}{2}\left(r r_{0}^{2}+r^{3}+r_{0}^{3}-r_{0} r^{2}\right)\right|^{2}+2\left|r-\frac{1}{2}\left(r r_{0}^{2}+r^{3}-r_{0}^{3}+r_{0} r^{2}\right)\right|^{2} \\
& \left.+\left|1-2 r_{0}+r_{0}^{2}\right|^{2}+\left|1-2 r+r^{2}\right|^{2}\right] .
\end{aligned}
$$

Here $f_{1}=\frac{1}{16}\left[2 r^{2}+2\left(r^{2} r_{0}+r^{3}-r_{0}^{3}+r_{0}^{2} r\right)+r^{4}-r_{0}^{4}+2 r^{2} r_{0}^{2}\right]$ and $f_{2}=\frac{1}{8}\left(r_{0}-1\right)^{2}$. The fidelity and the efficiency of our P-P-SWAP gate varying with the parameter $g / \sqrt{\kappa \gamma}$ are shown in Figs. 11(a) and 11(b), respectively.

[1] M. A. Nielsen and I. L. Chuang, Quantum computation and quantum information (Cambridge University Press, 
[2] C. H. Bennett, G. Brassard, C. Crepeau, R. Jozsa, A. Peres, and W. K. Wootters, Teleporting an unknown quantum state via dual classical and Einstein-PodolskyRosen channels, Phys. Rev. Lett. 70, 1895 (1993).

[3] C. H. Bennett and S. J. Wiesner, Communication via one- and two-particle operators on Einstein-PodolskyRosen states, Phys. Rev. Lett. 69, 2881-2884 (1992).

[4] X. S. Liu, G. L. Long, D. M. Tong, and F.Li, General scheme for superdense coding between multiparties, Phys. Rev. A 65, 022304 (2002).

[5] A. K. Ekert, Quantum cryptography based on Bell's theorem. Phys. Rev. Lett. 67, 661-663 (1991).

[6] C. H. Bennett, G. Brassard, and N. D. Mermin, Quantum cryptography without Bell's theorem. Phys. Rev. Lett. 68, 557-559 (1992).

[7] M. Hillery, V. Bužek, and A. Berthiaume, Quantum secret sharing, Phys. Rev. A 59, 1829 (1999).

[8] G. L. Long and X. S. Liu, Theoretically efficient highcapacity quantum-key-distribution scheme, Phys. Rev. A 65, 032302 (2002).

[9] F. G. Deng, G. L. Long, and X. S. Liu, Two-step quantum direct communication protocol using the EinsteinPodolsky-Rosen pair block, Phys. Rev. A 68, 042317 (2003).

[10] J. T. Barreiro, T. C. Wei, and P. G. Kwiat, Beating the channel capacity limit for linear photonic superdense coding, Nat. Phys. 4, 282 (2008).

[11] P. G. Kwiat and H. Weinfurter, Embedded Bell-state analysis, Phys. Rev. A 58, R2623-R2626 (1998).

[12] S. P. Walborn, S. Pádua, and C. H. Monken, Hyperentanglement-assisted Bell-state analysis, Phys. Rev. A 68, 042313 (2003).

[13] C. Schuck, G. Huber, C. Kurtsiefer, and H. Weinfurter, Complete deterministic linear optics Bell state analysis, Phys. Rev. Lett. 96, 190501 (2006).

[14] M. Barbieri, G. Vallone, P. Mataloni, and F. De Martini, Complete and detrministic deicrimination of polarization Bell states assisted by momentum entanglement, Phys. Rev. A 75, 042317 (2007).

[15] J. T. Barreiro, N. K. Langford, N. A. Peters, and P. G. Kwiat, Generation of hyperentangled photon pairs, Phys. Rev. Lett. 95, 260501 (2005).

[16] C. Cinelli, M. Barbieri, F. De Martini, and P. Mataloni, Realization of hyperentangled two-photon states, Laser Phys. 15, 124 (2005).

[17] M. Barbieri, C. Cinelli, F. De Martini, and P. Mataloni, Generation of $(2 \times 2)$ and $(4 \times 4)$ two-photon states with tunable degree of entanglement and mixedness, Fortschr. Phys. 52, 1102 (2004).

[18] M. Barbieri, C. Cinelli, P. Mataloni, and F. DeMartini, Polarization-momentum hyperentangled states: Realization and characterization, Phys. Rev. A 72, 052110 (2005).

[19] D. Bhatti, J. von Zanthier, and G. S. Agarwal, Entanglement of polarization and orbital angular momentum, Phys. Rev. A 91, 062303 (2015).

[20] A. Rossi, G. Vallone, A. Chiuri, F. De Martini, and P. Mataloni, Mulipath entanglement of two photons, Phys. Rev. Lett. 102, 153902 (2009).

[21] W. B. Gao, C. Y. Lu, X. C. Yao, P. Xu, O. Guhne, A. Goebel, Y. A. Chen, C. Z. Peng, Z. B. Chen, and J. W. Pan, Experimental demonstration of a hyperentangled ten-qubit Schrödinger cat state, Nat. Phys. 6, 331 (2010).

[22] T. J. Wang, Y. Lu, and G. L. Long, Generation and complete analysis of the hyperentangled Bell state for photons assisted by quantum-dot spins in optical microcavities, Phys. Rev. A 86, 042337 (2012).

[23] G. Vallone, R. Ceccarelli, F. De Martini, and P. Mataloni, Hyperentanglement of two photons in three degrees of freedom, Phys. Rev. A 79, 030301(R) (2009).

[24] C. H. Bennett, G. Brassard, S. Popescu, B. Schumacher, J. A. Smolin, and W. K. Wootters, Purification of noise entanglement and faithful teleportation via noisy channels, Phys. Rev. Lett. 76, 722-725 (1996).

[25] D. Deutsch, A. Ekert, R. Jozsa, C. Macchiavello, S. Popescu, and A. Sanpera, Quantum privacy amplification and the security of quantum cryptography over noisy channel, Phys. Rev. Lett. 77, 2818-2821 (1996).

[26] J. W. Pan, C. Simon, $\breve{C}$. Brukner, and A. Zeilinger, Entanglement purification for quantum communication, Nature (London) 410, 1067-1070 (2001).

[27] C. Simon and J. W. Pan, Polarization entanglement purification using spatial entanglement, Phys. Rev. Lett. 89, 257901 (2002).

[28] Y. B. Sheng, F. G. Deng, and H. Y. Zhou, Efficient polarization-entanglement purification based on parametric down-conversion sources with cross-Kerr nonlinearity, Phys. Rev. A 77, 042308 (2008).

[29] R. F. Werner, Quantum states with Einstein-PodolskyRosen correlations admitting a hidden-variable model, Phys. Rev. A 40, 4277 (1989).

[30] Y. B. Sheng and F. G. Deng, Deterministic entanglement purification and complete nonlocal Bell-state analysis with hyperentangledment, Phys. Rev. A 81, 032307 (2010).

[31] Y. B. Sheng and F. G. Deng, One-step deterministic polarization-entanglement purification using spatial entanglement, Phys. Rev. A 82, 044305 (2010)

[32] X. H. Li, Deterministic polarization-entanglement purification using spatial entanglement, Phys. Rev. A 82, 044304 (2010).

[33] F. G. Deng, One-step error correction for multipartite polarization entanglement, Phys. Rev. A 83, 062316 (2011).

[34] Y. B. Sheng and L. Zhou, Deterministic polarization entanglement purification using time-bin entanglement, Laser Phys. Lett. 11, 085203 (2014).

[35] J. W. Pan, S. Gasparoni, R. Ursin, G. Weihs, and A. Zeilinger, Experimental entanglement purification of arbitrary unknown states, Nature (London) 423, 417-422 (2003).

[36] B. C. Ren and F. G. Deng, Hyperentanglement purification and concentration assisted by diamond NV centers inside photoic crystal cavities, Laser Phys. Lett. 10, 115201 (2013); B. C. Ren, F. F. Du, and F. G. Deng, Two-step hyperentanglement purification with the quantum-state-joining method, Phys. Rev. A 90, 052309 (2014).

[37] G. Balasubramanian, P. Neumann, D. Twitchen, M. Markham, R. Kolesov, N. Mizuochi, J. Isoya, J. Achard, J. Beck, J. Tissler, V. Jacques, P. R. Hemmer, F. Jelezko, and J. Wrachtrup, Ultralong spin coherence time in isotopically engineered diamond, Nat. Mater. 8, 383-387 (2009).

[38] F. Jelezko, T. Gaebel, I. Popa, A. Gruber, and J. Wrachtrup, Obzervation of coherent oscillations in a single electron spin, Phys. Rev. Lett. 92, 076401 (2004).

[39] T. Gaebel, M. Domhan, I. Popa, C. Wittmann, P. Neu- 
mann, F. Jelezko, J. R. Rabeau, N. Stavrias, A. D. Greentree, S. Prawer, J. Meijer, J. Twamley, P. R. Hemmer, and J. Wrachtrup, Room-temperature coherent coupling of single spins in diamond, Nat. Phys. 2, 408-413 (2006).

[40] G. D. Fuchs, V. V. Dobrovitski, D. M. Toyli, F. J. Heremans, D. D. Awschalom, Gigahertz dynamics of a strongly driven single quantum spin, Science 326, 15021522 (2009).

[41] B. B. Buckley, G. D. Fuchs, L. C. Bassett, and D. D. Awschalom, Spin-light coherence for single-spin measurement and control in diamond, Science 330, 1212-1215 (2010).

[42] L. Jian, J. S. Hodges, J. R. Maze, P. Maurer, J. M. Taylor, D. G. Cory, P. R. Hemmer, R. L. Walsworth, A. Yacoby, A. S. Zibrov, and M. D. Lukin, Repetitive readout of a single electronic spin via quantum logic with nuclear spin ancillae, Science 326, 267-272 (2009).

[43] L. Robledo, L. Chidress, H. Bernien, B. Hensen, P. F. A. Alkemade, and R. Hanson, High-fidelity projective read-out of a solid-state spin quantum register, Nature (London) 477, 574-578 (2011).

[44] A. Young, C. Y. Hu, L. Marseglia, J. P. Harrison, J. L. O'Brien, and J. G. Rarity, Cavity enhanced spin measurement of the ground state spin of an NV center in diamond, New J. Phys. 11, 013007 (2009).

[45] Q. Chen, W. Yang, M. Feng, and J. F. Du, Entangling separate nitrogen-vacancy centers in a scalable fashion via coupling to microtoroidal resonators, Phys. Rev. A 83, 054305 (2011).

[46] A. Zheng, J. Li, R. Yu, X. Y. Lü, and Y. Wu, Generation of Greenberger-Horne-Zeilinger state of distant diamond nitrogen-vacancy centers via nanocavity inputoutput process, Opt. Express 20, 16902 (2012).

[47] H. R. Wei and G. L. Long, Hybrid quantum gates between flying photon and diamond nitrogen-vacancy centers assisted by optical microcavities, Sci. Rep. 5, 12918 (2015).

[48] Q. Liu and M. Zhang, Generation and complete nondestructive analysis of hyperentanglement assisted by nitrogen-vacancy centers in resonators, Phys. Rev. A 91, 062321 (2015).

[49] P. E. Barclay, K. M. Fu, C. Santori, and R. G. Beausoleil, Chip-based microcavities coupled to nitrogen-vacancy centers in single crystal diamond, Appl. Phys. Lett. 95, 191115 (2009).

[50] Y. S. Park, A. K. Cook, and H. Wang, Cavity QED with diamond nanocrystal and silica microspheres, Nano Lett. 6, 2075 (2006).

[51] D. Englund, B. Shields, K. Rivoire, Fariba Hatami, J. Vučković, H. Park, and M. D. Lukin, Deterministic coupling of a single nitrogen vacancy center to a photoic crystal cavity, Nano Lett. 10, 3922 (2010).
[52] J. Wolters, A. W. Schell, Güsse, M. Schoengen, H. Döscher, T. Hannappel, B. Löchel, M. Barth, and O. Benson, Enhancement of the zero phonon line emission from a single nitrogen vacancy center in a nanodiamond via coupling to a photonic crystal cavity, Appl. Phys. Lett. 97, 141108 (2010).

[53] O. Arcizet, V. Jacques, A. Siria, P. Poncharal, P. Vincent, and S. Seidelin, A single nitrogen-vacancy defect coupled to a nanomechanical oscillator, Nat. Phys. 7, 879 (2011).

[54] D. F. Walls and G. J. Milburn, Quantum optics, (Springer-Verleg, Berlin, 1994).

[55] J. H. An, M. Feng, and C. H. Oh, Quantum-information processing with a single photon by an input-output process with respect to low-Q cavities, Phys. Rev. A 79, 032303 (2009).

[56] C. Y. Hu, A. Young, J. L. OBrien, W. J. Munro, and J. G. Rarity, Giant optical Faraday rotation induced by a single-electron spin in a quantum dot: Applications to entangling remote spins via a single photon, Phys. Rev. B 78, 085307 (2008).

[57] I. J. Luxmoore, E. D. Ahmadi, B. J. Luxmoore, N. A. Wasley, A. I. Tartakovskii, M. Hugues, M. S. Skolnick, and A. M. Fox, Restoring mode degeneracy in H1 photonic crystal cavities by uniaxial strain tuning, Appl. Phys. Lett. 100, 121116 (2012).

[58] J. Gudat, C. Bonato, E. van Nieuwenburg, S. Thon, H. Kim, P. M. Petroff, M. P. van Exter, and Dirk Bouweester, Permanent tuning of quantum dot transitions to degenerate microcavity resonances, Appl. Phys. Lett. 98, 12111 (2011).

[59] C. Bonato, D. Ding, J. Gudat, S. Thon, H. Kim, P. M. Petroff, M. P. van Exter, and D. Bouwmeester, Tuning micropillar cavity birefringence by laser inducd surface defects, Appl. Phys. Lett. 95, 251104 (2009).

[60] C. Bonato, E. van Nieuwenburg, J. Gudat, S. Thon, H. Kim, M. P. van Exter, and D. Bouwmeester, Strain tuning of quantum dot optical transtions via laser-induced surface defects, Phys. Rev. B 84, 075306 (2011).

[61] J. Hagemeier, C. Bonato, T. A. Truong, H. Kim, G. J. Beirne, M. Bakker, M. P. van Exter, Y. Q. Luo, P. Petroff, and D. Bouwmeester, H1 photonic crystal cavities for hybrid quantum information protocols, Opt. Express 20, 24714-24726 (2012).

[62] K. Hennessy, C. Högerle, E. Hu, A. Badolato, and A. Imamo $\breve{g l u}$, Tuning photonic nanocavities by atomic force microscope nano-oxidation, Appl. Phys. Lett. 89, 041118 (2006).

[63] Y. Eto, A. Noguchi, P. Zhang, M. Ueda, and M. Kozuma, Projective measurement of a single nuclear spin qubit by using two-mode cavity QED, Phys. Rev. Lett. 106, 160501 (2011). 